\title{
DUBROVIN EQUATIONS AND INTEGRABLE SYSTEMS ON HYPERELLIPTIC CURVES
}

\author{
FRITZ GESZTESY and HELGE HOLDEN*
}

\begin{abstract}
We introduce the most general version of Dubrovin-type equations for divisors on a hyperelliptic curve $\mathscr{K}_{\mathfrak{g}}$ of arbitrary genus $\mathfrak{g} \in \mathrm{N}$, and provide a new argument for linearizing the corresponding completely integrable flows. Detailed applications to completely integrable systems, including the KdV, AKNS, Toda, and the combined sine-Gordon and $\mathrm{mKdV}$ hierarchies, are made. These investigations uncover a new principle for $1+1$-dimensional integrable soliton equations in the sense that the Dubrovin equations, combined with appropriate trace formulas, encode all hierarchies of soliton equations associated with hyperelliptic curves. In other words, completely integrable hierarchies of soliton equations determine Dubrovin equations and associated trace formulas and, vice versa, Dubrovin-type equations combined with trace formulas permit the construction of hierarchies of soliton equations.
\end{abstract}

\section{Introduction}

The purpose of this paper is to re-examine Dubrovin equations for divisors on hyperelliptic Riemann surfaces and to underscore their exceptional role in connection with completely integrable hierarchies of soliton equations.

Starting from four representative hierarchies, the Korteweg-de Vries (KdV), Ablowitz-Kaup-Newell-Segur (AKNS), Toda lattice (Tl), and the combined sine-Gordon and $\mathrm{mKdV}(\mathrm{sGmKdV})$ hierarchy, we derive a new argument for linearizing the corresponding completely integrable flows. As a result of these investigations we show that a proper combination of Dubrovin equations and trace formulas involving auxiliary divisors on hyperelliptic curves encodes all information on the underlying completely integrable hierarchy of soliton equations.

In Section 2 we briefly review some basic facts on hyperelliptic curves and establish the notation used throughout this paper. Section 3 provides a "crash course" into the four different hierarchies closely following the detailed accounts in [11], [28], [29], [30], and [31]. In particular, we outline an elementary polynomial, recursive approach to these hierarchies, as originally developed

\footnotetext{
* Research supported in part by the Research Council of Norway under grant 107510/410 and the University of Missouri Research Board grant RB-97-086.

Received May 20, 1999; in revised form December 7, 1999.
} 
by S. I. Al'ber [1], [2] in the KdV context, and introduce the corresponding divisors on $\mathscr{K}_{\mathfrak{g}}$ associated with their algebro-geometric solutions. Section 4 relates the polynomial recursion relation approach with elementary symmetric functions (and functions derived from them) of $\mu_{1}\left(x, t_{r}\right), \ldots, \mu_{\mathfrak{g}}\left(x, t_{r}\right)$, where $\mu_{j}\left(x, t_{r}\right)$ are certain analogs of Dirichlet-type eigenvalues of the corresponding Lax operator. In Section 5 we study Dubrovin equations and, based on the results of Section 4, provide a new proof of the fundamental fact that a change of coordinates effected by the Abel map straightens out the flows on the Jacobi variety $J\left(\mathscr{K}_{\mathrm{g}}\right)$ of $\mathscr{K}_{\mathrm{g}}$. Section 6 illustrates our results by deriving connections between the KdV and sG equations, and AKNS and Toda hierarchies, respectively. These connections establish the fundamental role played by the Dubrovin equations as the common underlying principle for hierarchies of soliton equations. In particular, our formalism establishes an isomorphism between the class of algebro-geometric solutions of these integrable systems. Finally, Appendix A collects some useful results in connection with Lagrange interpolation formulas.

We emphasize that our results are not necessarily restricted to hyperelliptic curves. In particular, the approach of this paper applies to Boussinesq-type curves using the polynomial recursion formalism for the Boussinesq hierarchy developed in [19], [20].

Depending perhaps a bit on one's taste, the results of this paper may at first sight appear somewhat discouraging as they clearly shift the emphasis from individual hierarchies of soliton equations toward Dubrovin-type equations. On the positive side, however, they establish the Dubrovin equations as a universal object underlying all hierarchies.

We note that our approach to completely integrable soliton equations is close in spirit to that developed by M. S. Al'ber and S. I. Al'ber in a series of papers (see, e.g., [1]-[7] and the references therein). While their approach focuses on algebraically integrable systems and hence on a Hamiltonian formalism with associated action and angle variables, our approach concentrates on how a combination of elementary symmetric functions of $\mu_{1}\left(x, t_{1}\right), \ldots, \mu_{\mathfrak{g}}\left(x, t_{r}\right)$ and certain trace formulas generate completely integrable hierarchies of soliton equations and their algebro-geometric solutions.

Finally, we stress that the use of elementary symmetric functions and hence of trace formulas in terms of Dirichlet eigenvalues has a long history in the context of integrable equations. In fact, as early as 1975, Flaschka [25] characterized the real-valued periodic potentials $q$ with finitely many stability intervals of the associated Schrödinger operator $-d^{2} / d x^{2}+q$ in $L^{2}(\mathrm{R} ; d x)$ as stationary solutions of the KdV hierarchy using (regularized) trace relations for Dirichlet eigenvalues associated with $q$ and the underlying periodicity interval. 


\section{Hyperelliptic curves}

Fix $N \in \mathrm{N}_{0}$. We briefly review hyperelliptic Riemann surfaces of the type,

$$
\begin{aligned}
& \mathscr{F}_{N}(z, y)=y^{2}-R_{N+1}(z)=0, \quad R_{N+1}(z)=\prod_{m=0}^{N}\left(z-E_{m}\right), \\
& \left\{E_{m}\right\}_{m=0, \ldots, N} \subset \mathrm{C}, \quad E_{m} \neq E_{m^{\prime}} \text { for } m \neq m^{\prime} .
\end{aligned}
$$

The material of this section is standard and can be found, for instance, in [24]. The curve (2.1) is compactified by adding one point $P_{\infty}$ at infinity if $N$ is even, and two points $P_{\infty_{+}}$and $P_{\infty_{-}}, P_{\infty_{+}} \neq P_{\infty_{-}}$if $N$ is odd.

One then introduces an appropriate set of ${ }^{1}\lfloor N+1\rfloor / 2$ nonintersecting cuts $\mathscr{C}_{j}$ joining $E_{m(j)}$ and $E_{m^{\prime}(j)}$ and $\mathscr{C}_{\infty}$ joining $E_{N}$ and $\infty$ if $N$ is even. We denote

$$
\mathscr{C}=\bigcup_{j \in J \cup\{\infty\}} \mathscr{C}_{j}, \quad \mathscr{C}_{j} \cap \mathscr{C}_{k}=\emptyset, \quad j \neq k,
$$

where $J \subseteq\{1, \ldots,\lfloor N+1\rfloor / 2\}$. Defining the cut plane

$$
\Pi=C \backslash \mathscr{C},
$$

and then introduces the holomorphic function

$$
R_{N+1}(\cdot)^{1 / 2}: \Pi \rightarrow \mathrm{C}, \quad z \mapsto\left(\prod_{m=0}^{N}\left(z-E_{m}\right)\right)^{1 / 2}
$$

on $\Pi$ with an appropriate choice of the square root branch in (2.4). Next one defines

$$
\mathscr{M}_{\mathrm{g}}=\left\{\left(z, \sigma R_{N+1}(z)^{1 / 2}\right) \mid z \in \mathrm{C}, \sigma \in\{ \pm 1\}\right\} \cup \begin{cases}\left\{P_{\infty}\right\} & \text { for } N \text { even, } \\ \left\{P_{\infty_{+}}, P_{\infty_{-}}\right\} & \text {for } N \text { odd, }\end{cases}
$$

by extending $R_{N+1}(\cdot)^{1 / 2}$ to $\mathscr{C}$. The hyperelliptic curve $\mathscr{K}_{\mathrm{g}}$ is then the set $\mathscr{M}_{\mathrm{g}}$ with its natural complex structure obtained upon gluing the two sheets of $\mathscr{M}_{\mathrm{g}}$ crosswise along the cuts. Finite points $P$ on $\mathscr{K}_{\mathfrak{g}}$ are denoted by $P=(z, y)$, where $y(\cdot)$ denotes the meromorphic function on $\mathscr{K}_{\mathfrak{g}}$ satisfying $\mathscr{F}_{N}(z, y)=$ $y^{2}-R_{N+1}(z)=0 ; \mathscr{K}_{\mathfrak{g}}$ has genus $\mathfrak{g}=\lfloor N+1\rfloor / 2$.

A basis of $g$ linearly independent holomorphic differentials on $\mathscr{K}_{\mathfrak{g}}$ is given by $z^{\ell-1} d z / y$ for $\ell=1, \ldots, \mathfrak{g}$, and one introduces

$$
d \omega_{j}(P)=\sum_{\ell=1}^{\mathfrak{g}} c_{j, \ell} \frac{z^{\ell-1} d z}{y}, \quad j=1, \ldots, \mathfrak{g},
$$

\footnotetext{
${ }^{1}\lfloor x\rfloor=\sup \{y \in Z \mid y \leq x\}$.
} 
with normalization,

$$
\int_{a_{j}} d \omega_{k}=\delta_{j, k}, \quad j, k=1, \ldots, \mathfrak{g},
$$

where $\left\{a_{j}, b_{j}\right\}_{j=1}^{\mathfrak{g}}$ is a homology basis for $\mathscr{K}_{\mathfrak{g}}$.

Defining the matrix $\tau=\left(\tau_{j, k}\right)$ by

$$
\tau_{j, k}=\int_{b_{j}} d \omega_{k}, \quad j, k=1, \ldots, \mathfrak{g},
$$

one infers $\operatorname{Im}(\tau)>0$ and $\tau_{j, k}=\tau_{k, j}$.

We fix a base point $P_{0}$ on $\mathscr{K}_{\mathfrak{g}}$ and define the Abel map $\underline{A}_{P_{0}}$ by

$$
\underline{A}_{P_{0}}(P)=\left(\int_{P_{0}}^{P} d \omega_{1}, \ldots, \int_{P_{0}}^{P} d \omega_{\mathfrak{g}}\right) \quad\left(\bmod L_{\mathfrak{g}}\right), \quad P \in \mathscr{K}_{\mathrm{g}},
$$

with period lattice

$$
L_{\mathfrak{g}}=\left\{\underline{n}+\tau \underline{m} \mid \underline{n}, \underline{m} \in Z^{\mathfrak{g}}\right\} .
$$

Similarly, one introduces

$$
\underline{\alpha}_{P_{0}}: \operatorname{Div}\left(\mathscr{K}_{\mathfrak{g}}\right) \rightarrow J\left(\mathscr{K}_{\mathfrak{g}}\right), \quad \mathscr{D} \mapsto \underline{\alpha}_{P_{0}}(\mathscr{D})=\sum_{P \in \mathscr{K}_{\mathfrak{g}}} \mathscr{D}(P) \underline{A}_{P_{0}}(P)
$$

where $\operatorname{Div}\left(\mathscr{K}_{\mathfrak{g}}\right)$ and $J\left(\mathscr{K}_{\mathfrak{g}}\right)=\mathrm{C}^{\mathfrak{g}} / L_{\mathfrak{g}}$ denote the set of divisors on $\mathscr{K}_{\mathfrak{g}}$ and the Jacobi variety of $\mathscr{K}_{\mathfrak{g}}$, respectively.

In connection with divisors on $\mathscr{K}_{\mathfrak{g}}$ we shall employ the following (additive) notation,

$$
\begin{aligned}
\mathscr{D}_{Q_{0} \underline{Q}}=\mathscr{D}_{Q_{0}}+\mathscr{D}_{\underline{Q}}, \quad \mathscr{D}_{\underline{Q}}=\mathscr{D}_{Q_{1}}+\cdots+\mathscr{D}_{Q_{n}}, \text { etc. }, \\
\text { for } \underline{Q}=\left(Q_{1}, \ldots, Q_{n}\right) \in \sigma^{n} \mathscr{K}_{\mathfrak{g}},
\end{aligned}
$$

where for any $Q \in \mathscr{K}_{\mathfrak{g}}$,

$$
\mathscr{D}_{Q}: \mathscr{K}_{\mathfrak{g}} \rightarrow \mathrm{N}_{0}, \quad P \mapsto \mathscr{D}_{Q}(P)= \begin{cases}1 & \text { for } P=Q, \\ 0 & \text { for } P \in \mathscr{K}_{\mathfrak{g}} \backslash\{Q\},\end{cases}
$$

and $\sigma^{n} \mathscr{K}_{\mathfrak{g}}$ denotes the $n$th symmetric product of $\mathscr{K}_{\mathfrak{g}}$. 


\section{The hierarchies}

We give a brief presentation of the KdV, AKNS, sGmKdV, and Toda hierarchies based on a polynomial, recursive approach. The material of this section originated with work of S. I. Al'ber [1], [2] (see also [3]-[7]). It has been further developed in [11], [28], [29], [30], and [31] and we closely follow the latter sources. Common to all these hierarchies is that one can naturally associate with each one of them a hyperelliptic curve as described in the previous section.

In the following all coefficients of differential expressions are assumed to be smooth (or meromorphic) with respect to $x \in \mathrm{R}$ (or $x \in \mathrm{C}$ ) and $C^{1}(\mathrm{R})$ with respect to $t_{\mathfrak{g}}$.

The KdV hierarchy. The Lax pair consists of a second-order linear differential expression $L$ of Schrödinger-type,

$$
L\left(t_{\mathfrak{g}}\right)=-\frac{d^{2}}{d x^{2}}+V\left(x, t_{\mathfrak{g}}\right), \quad\left(x, t_{\mathfrak{g}}\right) \in \mathrm{R}^{2},
$$

and a differential expression $P_{2 \mathfrak{g}+1}\left(t_{\mathfrak{g}}\right)$ of order $2 \mathfrak{g}+1$ defined recursively as follows. Let $\left\{f_{j}\right\}_{j \in \mathrm{N}_{0}}$ be given by

$$
f_{0}=1, \quad f_{j, x}=-\frac{1}{4} f_{j-1, x x x}+V f_{j-1, x}+\frac{1}{2} V_{x} f_{j-1}, \quad j \in \mathrm{N} .
$$

Next, one defines

$$
P_{2 \mathfrak{g}+1}\left(t_{\mathfrak{g}}\right)=\sum_{j=0}^{\mathfrak{g}}\left(f_{j}\left(t_{\mathfrak{g}}\right) \frac{d}{d x}-\frac{1}{2} f_{j, x}\left(t_{\mathfrak{g}}\right)\right) L\left(t_{\mathfrak{g}}\right)^{\mathfrak{g}-j}, \quad \mathfrak{g} \in \mathrm{N}_{0},
$$

and using the definition of $f_{j}$ in (3.2), one finds that the commutator of $L\left(t_{\mathrm{g}}\right)$ and $P_{2 \mathfrak{g}+1}\left(t_{\mathfrak{g}}\right)$ is in fact a multiplication operator. Indeed, the Lax commutator representation reads

$$
L_{t_{\mathfrak{g}}}\left(t_{\mathfrak{g}}\right)-\left[P_{2 \mathfrak{g}+1}\left(t_{\mathfrak{g}}\right), L\left(t_{\mathfrak{g}}\right)\right]=V_{t_{\mathfrak{g}}}-2 f_{\mathfrak{g}+1, x}\left(x, t_{\mathfrak{g}}\right)=\operatorname{KdV}_{\mathfrak{g}}(V)=0 .
$$

Next define the polynomial ${ }^{2}$ of degree $\mathfrak{g}$ in $z$

$$
F_{\mathfrak{g}}\left(z, x, t_{\mathfrak{g}}\right)=\sum_{j=0}^{\mathfrak{g}} f_{\mathfrak{g}-j}\left(x, t_{\mathfrak{g}}\right) z^{j}=\prod_{j=1}^{\mathfrak{g}}\left(z-\mu_{j}\left(x, t_{\mathfrak{g}}\right)\right)
$$

\footnotetext{
${ }^{2}$ The zeros $\mu_{j}\left(x, t_{\mathfrak{g}}\right)$ of $F_{\mathfrak{g}}$ turn out to be eigenvalues associated with $L\left(t_{\mathfrak{g}}\right)$ and a Dirichlet boundary condition at the point $x \in \mathrm{R}$.
} 
implying

$$
-2 V_{t_{\mathrm{g}}}=F_{\mathrm{g}, x x x}-4(V-z) F_{\mathrm{g}, x}-2 V_{x} F_{\mathrm{g}} .
$$

In the special stationary case, defined by $V_{t_{\mathrm{g}}}=0$, this integrates to

$$
\frac{1}{2} F_{\mathfrak{g}, x x} F_{\mathfrak{g}}-\frac{1}{4} F_{\mathfrak{g}, x}^{2}-(V-z) F_{\mathfrak{g}}^{2}=R_{2 \mathfrak{g}+1}(z) .
$$

Here $R_{2 \mathfrak{g}+1}$ is a monic polynomial of degree $2 \mathfrak{g}+1$ with zeros $\left\{E_{0}, \ldots, E_{2 \mathfrak{g}}\right\}$. Hence,

$$
R_{2 \mathfrak{g}+1}(z)=\prod_{m=0}^{2 \mathfrak{g}}\left(z-E_{m}\right), \quad\left\{E_{m}\right\}_{m=0, \ldots, 2 \mathfrak{g}} \subset \mathrm{C} .
$$

The hyperelliptic curve $\mathscr{K}_{\mathrm{g}}$ is defined in terms of the stationary KdV hierarchy obtained by considering a $t_{\mathfrak{g}}$-independent function $V=V(x)$, resulting in

$$
\left[P_{2 \mathfrak{g}+1}, L\right]=2 f_{\mathfrak{g}+1, x}=0 .
$$

The classical Burchnall-Chaundy theorem [12], [13], [14] (see also [39], [42]) states that commuting differential operators are algebraically related. In the present context one finds

$$
P_{2 \mathfrak{g}+1}^{2}=R_{2 \mathfrak{g}+1}(L)
$$

and thus the hyperelliptic curve $\mathscr{K}_{\mathfrak{g}}$ of genus $\mathfrak{g}$ is of the type $y^{2}=R_{2 \mathfrak{g}+1}(z)$, with $N=2 \mathrm{~g}$ even when compared to Section 2 .

For later purpose we quote the following asymptotic high-energy expan$\operatorname{sion}^{3}$ (see [26], [31], [40])

$$
\frac{i F_{\mathfrak{g}}\left(z, x, t_{\mathfrak{g}}\right)}{2 R_{2 \mathfrak{g}+1}(z)^{1 / 2}} \underset{z \rightarrow \infty}{=} \frac{i}{2 z^{1 / 2}} \sum_{j=0}^{\infty} \hat{f}_{j}\left(x, t_{\mathfrak{g}}\right) z^{-j},
$$

where $\hat{f}_{j}$ denotes the homogeneous coefficients $f_{j}$ in (3.2) with all integration constants put equal to zero.

We also introduce the following fundamental meromorphic function $\phi\left(\cdot, x, t_{\mathfrak{g}}\right)$ on $\mathscr{K}_{\mathrm{g}}$,

$$
\phi\left(P, x, t_{\mathfrak{g}}\right)=\frac{i y+\frac{1}{2} F_{\mathfrak{g}, x}\left(z, x, t_{\mathfrak{g}}\right)}{F_{\mathfrak{g}}\left(z, x, t_{\mathfrak{g}}\right)}=\frac{-H_{\mathfrak{g}+1}\left(z, x, t_{\mathfrak{g}}\right)}{i y-\frac{1}{2} F_{\mathfrak{g}, x}\left(z, x, t_{\mathfrak{g}}\right)}
$$

\footnotetext{
${ }^{3}$ For an appropriate choice of the sign of the square roots in (3.11), the left-hand side of (3.11) equals the diagonal Green's function of $L\left(t_{\mathfrak{g}}\right)$.
} 
(the second equality in (3.12) serving as a definition of the polynomial $H_{\mathfrak{g}+1}$ of degree $g+1$ with respect to $z$ ), and the time-dependent Baker-Akhiezer function $\psi\left(\cdot, x, x_{0}, t_{\mathfrak{g}}, t_{0, \mathfrak{g}}\right)$ on $\mathscr{K}_{\mathfrak{g}} \backslash\left\{P_{\infty}\right\}$,

$$
\begin{aligned}
\psi\left(P, x, x_{0}, t_{\mathfrak{g}}, t_{0, \mathfrak{g}}\right)=\exp ( & \int_{t_{0, \mathfrak{g}}}^{t_{\mathfrak{g}}} d s\left(F_{\mathfrak{g}}\left(z, x_{0}, s\right) \phi\left(P, x_{0}, s\right)-\frac{1}{2} F_{\mathfrak{g}, x}\left(z, x_{0}, s\right)\right) \\
& \left.+\int_{x_{0}}^{x} d x^{\prime} \phi\left(P, x^{\prime}, t_{\mathfrak{g}}\right)\right) .
\end{aligned}
$$

The divisor $\left(\phi\left(\cdot, x, t_{\mathrm{g}}\right)\right)$ of $\phi\left(\cdot, x, t_{\mathrm{g}}\right)$ is given by

$$
\left(\phi\left(\cdot, x, t_{\mathfrak{g}}\right)\right)=\mathscr{D}_{\hat{v}_{0}\left(x, t_{\mathfrak{g}}\right) \underline{\hat{v}}\left(x, t_{\mathfrak{g}}\right)}-\mathscr{D} P_{\infty} \underline{\hat{\mu}}\left(x, t_{\mathfrak{g}}\right),
$$

where $\hat{\mu}\left(x, t_{\mathfrak{g}}\right)=\left(\hat{\mu}_{1}\left(x, t_{\mathfrak{g}}\right), \ldots, \hat{\mu}_{\mathfrak{g}}\left(x, t_{\mathfrak{g}}\right)\right) \in \sigma^{\mathfrak{g}} \mathscr{K}_{\mathfrak{g}}$,

$$
\hat{\mu}_{j}\left(x, t_{\mathfrak{g}}\right)=\left(\mu_{j}\left(x, t_{\mathfrak{g}}\right),-\frac{1}{2} F_{\mathfrak{g}, x}\left(\mu_{j}\left(x, t_{\mathfrak{g}}\right), x, t_{\mathfrak{g}}\right)\right), \quad j=1, \ldots, \mathfrak{g}
$$

denote the Dirichlet divisors ${ }^{2}$ and $\hat{v}_{0}\left(x, t_{\mathfrak{g}}\right) \underline{\hat{v}}\left(x, t_{\mathfrak{g}}\right)=\left(\hat{v}_{0}\left(x, t_{\mathfrak{g}}\right), \ldots, \hat{v}_{\mathfrak{g}}\left(x, t_{\mathfrak{g}}\right)\right) \in$ $\sigma^{\mathfrak{g}+1} \mathscr{K}_{\mathrm{g}}$,

$$
\hat{v}_{\ell}\left(x, t_{\mathfrak{g}}\right)=\left(v_{\ell}\left(x, t_{\mathfrak{g}}\right), \frac{1}{2} F_{\mathfrak{g}, x}\left(v_{\ell}\left(x, t_{\mathfrak{g}}\right), x, t_{\mathfrak{g}}\right)\right), \quad \ell=0, \ldots, \mathfrak{g}
$$

abbreviate the Neumann divisors ${ }^{4}$ derived from the zeros of $H_{\mathfrak{g}+1}\left(z, x, t_{\mathfrak{g}}\right)$,

$$
H_{\mathfrak{g}+1}\left(z, x, t_{\mathfrak{g}}\right)=\prod_{\ell=0}^{\mathfrak{g}}\left(z-v_{\ell}\left(x, t_{\mathfrak{g}}\right)\right) .
$$

The importance of $\phi$ in connection with divisors on hyperelliptic curves was recognized by Jacobi [33] and applied to the KdV case by Mumford [38], Sect. IIIa.1 and McKean [36] (see also [23], [39]).

The AKNS hierarchy. The Lax pair consists of a Dirac-type matrix-valued differential expression

$$
M\left(t_{\mathfrak{g}}\right)=i\left(\begin{array}{cc}
\frac{d}{d x} & -q\left(x, t_{\mathfrak{g}}\right) \\
p\left(x, t_{\mathfrak{g}}\right) & -\frac{d}{d x}
\end{array}\right), \quad\left(x, t_{\mathfrak{g}}\right) \in \mathrm{R}^{2},
$$

and a matrix-valued differential operator $Q_{\mathfrak{g}+1}\left(t_{\mathfrak{g}}\right)$ of order $\mathfrak{g}+1, \mathfrak{g} \in \mathrm{N}_{0}$. To define $Q_{\mathfrak{g}+1}\left(t_{\mathfrak{g}}\right)$ one proceeds as follows. Define $\left\{f_{\ell}\left(x, t_{\mathfrak{g}}\right)\right\}_{\ell \in \mathbb{N}_{0}},\left\{g_{\ell}\left(x, t_{\mathfrak{g}}\right)\right\}_{\ell \in \mathrm{N}_{0}}$,

\footnotetext{
${ }^{4}$ The zeros $v_{\ell}\left(x, t_{\mathfrak{g}}\right)$ of $H_{\mathfrak{g}+1}$ turn out to be eigenvalues associated with $L\left(t_{\mathfrak{g}}\right)$ and a Neumann boundary condition at the point $x \in \mathrm{R}$.
} 
and $\left\{h_{\ell}\left(x, t_{\mathfrak{g}}\right)\right\}_{\ell \in \mathrm{N}_{0}}$ recursively by,

$$
\begin{aligned}
f_{0} & =-i q, \\
f_{\ell+1} & =\frac{i}{2} f_{\ell, x}-i q g_{\ell+1}, \\
g_{0} & =1 \\
g_{\ell+1, x} & =p f_{\ell}+q h_{\ell}, \\
h_{0} & =i p \\
h_{\ell+1} & =-\frac{i}{2} h_{\ell, x}+i p g_{\ell+1}, \quad \ell \in \mathrm{N}_{0} .
\end{aligned}
$$

The $2 \times 2$ matrix $Q_{\mathfrak{g}+1}\left(t_{\mathfrak{g}}\right)$ is then defined by (3.20)

$$
Q_{\mathfrak{g}+1}\left(t_{\mathfrak{g}}\right)=i \sum_{\ell=0}^{\mathfrak{g}+1}\left(\begin{array}{cc}
-g_{\mathfrak{g}+1-\ell}\left(t_{\mathfrak{g}}\right) & f_{\mathfrak{g}-\ell}\left(t_{\mathfrak{g}}\right) \\
-h_{\mathfrak{g}-\ell}\left(t_{\mathfrak{g}}\right) & g_{\mathfrak{g}+1-\ell}\left(t_{\mathfrak{g}}\right)
\end{array}\right) M\left(t_{\mathfrak{g}}\right)^{\ell}, \quad \begin{aligned}
& \mathfrak{g} \in \mathrm{N}_{0}, \\
& f_{-1}=h_{-1}=0,
\end{aligned}
$$

and one verifies that the commutator of $Q_{\mathfrak{g}+1}\left(t_{\mathfrak{g}}\right)$ and $M\left(t_{\mathfrak{g}}\right)$ becomes

$$
\left[Q_{\mathfrak{g}+1}\left(t_{\mathfrak{g}}\right), M\left(t_{\mathfrak{g}}\right)\right]=\left(\begin{array}{cc}
0 & -2 i f_{\mathfrak{g}+1}\left(t_{\mathfrak{g}}\right) \\
2 i h_{\mathfrak{g}+1}\left(t_{\mathfrak{g}}\right) & 0
\end{array}\right) .
$$

Consequently, the Lax commutator representation for the AKNS hierarchy reads $\left(g \in N_{0}\right)$

$$
\begin{aligned}
\frac{d}{d t_{\mathfrak{g}}} M\left(t_{\mathfrak{g}}\right)-\left[Q_{\mathfrak{g}+1}\left(t_{\mathfrak{g}}\right), M\left(t_{\mathfrak{g}}\right)\right] & =\left(\begin{array}{c}
p_{t_{\mathfrak{g}}}\left(x, t_{\mathfrak{g}}\right)-2 h_{\mathfrak{g}+1}\left(x, t_{\mathfrak{g}}\right) \\
q_{t_{\mathfrak{g}}}\left(x, t_{\mathfrak{g}}\right)-2 f_{\mathfrak{g}+1}\left(x, t_{\mathfrak{g}}\right)
\end{array}\right) \\
& =\operatorname{AKNS}_{\mathfrak{g}}(p, q)=0 .
\end{aligned}
$$

Next, define polynomials $F_{\mathfrak{g}}, G_{\mathfrak{g}+1}$, and $H_{\mathfrak{g}}$ with respect to $z \in \mathrm{C}$,

$$
F_{\mathfrak{g}}\left(z, x, t_{\mathfrak{g}}\right)=\sum_{\ell=0}^{\mathfrak{g}} f_{\mathfrak{g}-\ell}\left(x, t_{\mathfrak{g}}\right) z^{\ell}=-i q\left(x, t_{\mathfrak{g}}\right) \prod_{j=1}^{\mathfrak{g}}\left(z-\mu_{j}\left(x, t_{\mathfrak{g}}\right)\right),
$$

$$
\begin{aligned}
G_{\mathfrak{g}+1}\left(z, x, t_{\mathfrak{g}}\right) & =\sum_{\ell=0}^{\mathfrak{g}+1} g_{\mathfrak{g}+1-\ell}\left(x, t_{\mathfrak{g}}\right) z^{\ell} \\
H_{\mathfrak{g}}\left(z, x, t_{\mathfrak{g}}\right) & =\sum_{\ell=0}^{\mathfrak{g}} h_{\mathfrak{g}-\ell}\left(x, t_{\mathfrak{g}}\right) z^{\ell}=i p\left(x, t_{\mathfrak{g}}\right) \prod_{j=1}^{\mathfrak{g}}\left(z-v_{j}\left(x, t_{\mathfrak{g}}\right)\right) .
\end{aligned}
$$


In the special stationary case, where $p_{t_{\mathrm{g}}}=q_{t_{\mathrm{g}}}=0$, one infers from the recursion (3.19) that $\left(G_{\mathfrak{g}+1}^{2}-F_{\mathfrak{g}} H_{\mathfrak{g}}\right)_{x}=0$ and hence

$$
G_{\mathfrak{g}+1}^{2}-F_{\mathfrak{g}} H_{\mathfrak{g}}=R_{2 \mathfrak{g}+2}(z),
$$

where $R_{2 \mathfrak{g}+2}$ is a monic polynomial of degree $2 \mathfrak{g}+2$ with zeros $\left\{E_{0}, \ldots, E_{2 \mathfrak{g}+1}\right\}$. Thus,

$$
R_{2 \mathfrak{g}+2}(z)=\prod_{m=0}^{2 \mathfrak{g}+1}\left(z-E_{m}\right), \quad\left\{E_{m}\right\}_{m=0, \ldots, 2 \mathfrak{g}+1} \subset \mathrm{C} .
$$

The stationary case determines the hyperelliptic curve $\mathscr{K}_{\mathfrak{g}}$ of genus $g$ of the type $y^{2}=R_{2 \mathfrak{g}+1}(z)$, with $N=2 \mathfrak{g}+1$ when compared to Section 2 . If $p=p(x)$ and $q=q(x)$ are stationary solutions of the AKNS equation,

$$
\left[Q_{\mathfrak{g}+1}, M\right]=0, \quad \text { that is, } f_{\mathfrak{g}+1}=h_{\mathfrak{g}+1}=0,
$$

Burchnall-Chaundy's theorem implies that

$$
Q_{\mathfrak{g}+1}^{2}+R_{2 \mathfrak{g}+2}(M)=0 .
$$

By studying the Green's matrix of $M$ one finds the following asymptotic high-energy expansion [30],

$$
\begin{aligned}
& \frac{F_{\mathfrak{g}}\left(z, x, t_{\mathfrak{g}}\right)}{R_{2 \mathfrak{g}+2}(z)^{1 / 2}} \underset{z \rightarrow \infty}{=} \frac{1}{z} \sum_{k=0}^{\infty} \hat{f}_{k}\left(x, t_{\mathfrak{g}}\right) z^{-k}, \\
& \frac{H_{\mathfrak{g}}\left(z, x, t_{\mathfrak{g}}\right)}{R_{2 \mathfrak{g}+2}(z)^{1 / 2}} \underset{z \rightarrow \infty}{=} \frac{1}{z} \sum_{k=0}^{\infty} \hat{h}_{k}\left(x, t_{\mathfrak{g}}\right) z^{-k}
\end{aligned}
$$

for an appropriate determination of the square roots in (3.28). Here $\hat{f}_{j}$ and $\hat{h}_{j}$ denote the homogeneous quantities with vanishing integration constants in (3.19).

We also record the meromorphic function $\phi\left(\cdot, x, t_{\mathfrak{g}}\right)$ on $\mathscr{K}_{\mathfrak{g}}$,

$$
\phi\left(P, x, t_{\mathfrak{g}}\right)=\frac{y+G_{\mathfrak{g}+1}\left(z, x, t_{\mathfrak{g}}\right)}{F_{\mathfrak{g}}\left(z, x, t_{\mathfrak{g}}\right)}=\frac{-H_{\mathfrak{g}}\left(z, x, t_{\mathfrak{g}}\right)}{y-G_{\mathfrak{g}+1}\left(z, x, t_{\mathfrak{g}}\right)},
$$

and the Baker-Akhiezer vector on $\mathscr{K}_{\mathfrak{g}} \backslash\left\{P_{\infty_{ \pm}}\right\}$,

$$
\Psi\left(P, x, x_{0}, t_{\mathfrak{g}}, t_{0, \mathfrak{g}}\right)=\left(\begin{array}{c}
\psi_{1}\left(P, x, x_{0}, t_{\mathfrak{g}}, t_{0, \mathfrak{g}}\right) \\
\psi_{2}\left(P, x, x_{0}, t_{\mathfrak{g}}, t_{0, \mathfrak{g}}\right)
\end{array}\right),
$$




$$
\begin{aligned}
\psi_{1}\left(P, x, x_{0}, t_{\mathfrak{g}}, t_{0, \mathfrak{g}}\right)= & \exp \left(\int_{x_{0}}^{x} d x^{\prime}\left(-i z+q\left(x^{\prime}, t_{\mathfrak{g}}\right) \phi\left(P, x^{\prime}, t_{\mathfrak{g}}\right)\right)\right. \\
& \left.+i \int_{t_{0, \mathfrak{g}}}^{t_{\mathfrak{g}}} d s\left(F_{\mathfrak{g}}\left(z, x_{0}, s\right) \phi\left(P, x_{0}, s\right)-G_{\mathfrak{g}+1}\left(z, x_{0}, s\right)\right)\right),
\end{aligned}
$$

$$
\psi_{2}\left(P, x, x_{0}, t_{\mathfrak{g}}, t_{0, \mathfrak{g}}\right)=\phi\left(P, x, t_{\mathfrak{g}}\right) \psi_{1}\left(P, x, x_{0}, t_{\mathfrak{g}}, t_{0, \mathfrak{g}}\right) .
$$

The divisor $\left(\phi\left(\cdot, x, t_{\mathfrak{g}}\right)\right)$ of $\phi\left(\cdot, x, t_{\mathfrak{g}}\right)$ is given by

$$
\left(\phi\left(\cdot, x, t_{\mathfrak{g}}\right)\right)=\mathscr{D}_{P_{\infty_{+}} \underline{\hat{v}}\left(x, t_{\mathfrak{g}}\right)}-\mathscr{D}_{P_{\infty_{-}} \underline{\hat{\mu}}\left(x, t_{\mathfrak{g}}\right)},
$$

where $\underline{\hat{\mu}}\left(x, t_{\mathfrak{g}}\right)=\left(\hat{\mu}_{1}\left(x, t_{\mathfrak{g}}\right), \ldots, \hat{\mu}_{\mathfrak{g}}\left(x, t_{\mathfrak{g}}\right)\right) \in \sigma^{\mathfrak{g}} \mathscr{K}_{\mathfrak{g}}$,

$$
\hat{\mu}_{j}\left(x, t_{\mathfrak{g}}\right)=\left(\mu_{j}\left(x, t_{\mathfrak{g}}\right), G_{\mathfrak{g}+1}\left(\mu_{j}\left(x, t_{\mathfrak{g}}\right), x, t_{\mathfrak{g}}\right)\right), \quad j=1, \ldots, \mathfrak{g}
$$

and $\underline{\hat{v}}\left(x, t_{\mathfrak{g}}\right)=\left(\hat{v}_{1}\left(x, t_{\mathfrak{g}}\right), \ldots, \hat{v}_{\mathfrak{g}}\left(x, t_{\mathfrak{g}}\right)\right) \in \sigma^{\mathfrak{g}} \mathscr{K}_{\mathfrak{g}}$,

$$
\hat{v}_{j}\left(x, t_{\mathfrak{g}}\right)=\left(v_{j}\left(x, t_{\mathfrak{g}}\right),-G_{\mathfrak{g}+1}\left(v_{j}\left(x, t_{\mathfrak{g}}\right), x, t_{\mathfrak{g}}\right)\right), \quad j=1, \ldots, \mathfrak{g} .
$$

The Toda hierarchy. Let $\left(S^{ \pm} f\right)(n)=f^{ \pm}(n)=f(n \pm 1), n \in \mathrm{Z}$ denote the shift operation on the lattice $Z$. The equations in the Toda hierarchy are continuous in time and discrete in the space variable. The Lax pair consists of the second-order difference operator

$$
L\left(t_{\mathfrak{g}}\right)=a\left(t_{\mathfrak{g}}\right) S^{+}+a^{-}\left(t_{\mathfrak{g}}\right) S^{-}-b\left(t_{\mathfrak{g}}\right), \quad t_{\mathfrak{g}} \in \mathrm{R}
$$

and a difference operator $P_{2 \mathfrak{g}+2}\left(t_{\mathfrak{g}}\right)$ of order $2 \mathfrak{g}+1, \mathfrak{g} \in \mathrm{N}_{0}$

$$
P_{2 \mathfrak{g}+2}\left(t_{\mathfrak{g}}\right)=-L\left(t_{\mathfrak{g}}\right)^{\mathfrak{g}+1}+\sum_{j=0}^{\mathfrak{g}}\left(g_{j}\left(t_{\mathfrak{g}}\right)+2 a\left(t_{\mathfrak{g}}\right) f_{j}\left(t_{\mathfrak{g}}\right) S^{+}\right) L\left(t_{\mathfrak{g}}\right)^{\mathfrak{g}-j}+f_{\mathfrak{g}+1}\left(t_{\mathfrak{g}}\right) .
$$

Here $\left\{f_{j}\left(n, t_{\mathrm{g}}\right)\right\}_{j \in \mathrm{N}_{0}}$ and $\left\{g_{j}\left(n, t_{\mathrm{g}}\right)\right\}_{j \in \mathrm{N}_{0}}$ satisfy the recursion relations,

$$
\begin{aligned}
& f_{0}=1, \quad g_{0}=-c_{1}, \\
& 2 f_{j+1}+g_{j}+g_{j}^{-}+2 b f_{j}=0, \quad j \in \mathrm{N}_{0}, \\
& g_{j+1}-g_{j+1}^{-}+2\left(a^{2} f_{j}^{+}-\left(a^{-}\right)^{2} f_{j}^{-}\right)+b\left(g_{j}-g_{j}^{-}\right)=0, \quad j \in \mathrm{N}_{0} .
\end{aligned}
$$

The Lax commutator representation of the Toda hierarchy then reads (3.38)

$$
L_{t_{\mathfrak{g}}}\left(t_{\mathfrak{g}}\right)-\left[P_{2 \mathfrak{g}+2}\left(t_{\mathfrak{g}}\right), L\left(t_{\mathfrak{g}}\right)\right]=\mathrm{Tl}_{\mathfrak{g}}(a, b)_{1} S^{+}-\mathrm{Tl}_{\mathfrak{g}}(a, b)_{2}+\mathrm{Tl}_{\mathfrak{g}}\left(a^{-}, b^{-}\right)_{1} S^{-}=0 \text {, }
$$


DUBROVIN EQUATIONS AND INTEGRABLE SYSTEMS ON HYPERELLIPTIC . . . 101

where

$$
\begin{aligned}
& \mathrm{Tl}_{\mathfrak{g}}(a, b)_{1}=a_{t_{\mathfrak{g}}}+a\left(g_{\mathfrak{g}}^{+}+g_{\mathfrak{g}}+f_{\mathfrak{g}+1}^{+}+f_{\mathfrak{g}+1}+2 b^{+} f_{\mathfrak{g}}^{+}\right)=0, \\
& \mathrm{Tl}_{\mathfrak{g}}(a, b)_{2}=b_{t_{\mathfrak{g}}}+2\left(b\left(g_{\mathfrak{g}}+f_{\mathfrak{g}+1}\right)+a^{2} f_{\mathfrak{g}}^{+}-\left(a^{-}\right)^{2} f_{\mathfrak{g}}^{-}+b^{2} f_{\mathfrak{g}}\right)=0 .
\end{aligned}
$$

This is equivalent to

$$
\mathrm{Tl}_{\mathfrak{g}}(a, b)=\left(\mathrm{Tl}_{\mathfrak{g}}(a, b)_{1}, \mathrm{Tl}_{\mathfrak{g}}(a, b)_{2}\right)^{t}=0, \quad \mathfrak{g} \in \mathrm{N}_{0}
$$

Next, define

$$
\begin{aligned}
F_{\mathfrak{g}}\left(z, n, t_{\mathfrak{g}}\right) & =\sum_{j=0}^{\mathfrak{g}} z^{j} f_{\mathfrak{g}-j}\left(n, t_{\mathfrak{g}}\right)=\prod_{j=1}^{\mathfrak{g}}\left(z-\mu_{j}\left(n, t_{\mathfrak{g}}\right)\right), \\
G_{\mathfrak{g}+1}\left(z, n, t_{\mathfrak{g}}\right) & =-z^{\mathfrak{g}+1}+\sum_{j=0}^{\mathfrak{g}} z^{j} g_{\mathfrak{g}-j}\left(n, t_{\mathfrak{g}}\right)+f_{\mathfrak{g}+1}\left(n, t_{\mathfrak{g}}\right) .
\end{aligned}
$$

In the special stationary case, defined by $a_{t_{\mathfrak{g}}}=b_{t_{\mathfrak{g}}}=0$, the recursion formulas (3.37) then imply

$$
G_{\mathfrak{g}+1}-4 a^{2} F_{\mathfrak{g}} F_{\mathfrak{g}}^{+}=G_{\mathfrak{g}+1}^{-}-4\left(a^{-}\right)^{2} F_{\mathfrak{g}}^{-} F_{\mathfrak{g}}=R_{2 \mathfrak{g}+2}(z),
$$

where $R_{2 \mathrm{~g}+2}(z)$ is a lattice constant. By inspection, $R_{2 \mathrm{~g}+2}(z)$ is a polynomial in $z$ of degree $2 \mathfrak{g}+2$ with zeros $\left\{E_{0}, \ldots, E_{2 \mathfrak{g}+1}\right\}$, that is,

$$
R_{2 \mathfrak{g}+2}(z)=\prod_{m=0}^{2 \mathfrak{g}+1}\left(z-E_{m}\right), \quad\left\{E_{m}\right\}_{m=0, \ldots, 2 \mathfrak{g}+1} \subset \mathrm{C} .
$$

Consider now the stationary hierarchy where $a=a(n)$ and $b=b(n)$ satisfy $\left[P_{2 \mathfrak{g}+2}, L\right]=0$, or

$$
\begin{array}{r}
g_{\mathfrak{g}}^{+}+g_{\mathfrak{g}}+f_{\mathfrak{g}+1}^{+}+f_{\mathfrak{g}+1}+2 b^{+} f_{\mathfrak{g}}^{+}=0, \\
b\left(g_{\mathfrak{g}}+f_{\mathfrak{g}+1}\right)+a^{2} f_{\mathfrak{g}}^{+}-\left(a^{-}\right)^{2} f_{\mathfrak{g}}^{-}+b^{2} f_{\mathfrak{g}}=0 .
\end{array}
$$

Burchnall-Chaundy's theorem then states that

$$
P_{2 \mathfrak{g}+2}^{2}=R_{2 \mathfrak{g}+2}(L) .
$$

Hence the hyperelliptic curve $\mathscr{K}_{\mathrm{g}}$ of genus $\mathrm{g}$ reads $y^{2}=R_{2 \mathrm{~g}+2}(z)$, and thus $N=2 \mathfrak{g}+1$ is odd in the terminology of Section 2 . 
Studying the diagonal Green's function of $L\left(t_{\mathfrak{g}}\right)$ yields the high-energy expansion [11]

$$
\frac{F_{\mathfrak{g}}\left(z, n, t_{\mathfrak{g}}\right)}{R_{2 \mathfrak{g}+2}(z)^{1 / 2}} \underset{z \rightarrow \infty}{=} \sum_{j=0}^{\infty} \hat{f}_{j}\left(n, t_{\mathfrak{g}}\right) z^{-j-1} \quad \text { for } \quad|z|>\|L\|,
$$

with an appropriate choice of the radical in (3.47). Here $\hat{f}_{j}$ and similarly $\hat{g}_{j}$, denote the homogeneous coefficients $f_{j}$ and $g_{j}$ with vanishing integration (actually, summation) constants in (3.37).

Furthermore, define the meromorphic function $\phi\left(\cdot, n, t_{\mathfrak{g}}\right)$ on $\mathscr{K}_{\mathfrak{g}}$ by

$$
\phi\left(P, n, t_{\mathfrak{g}}\right)=\frac{-G_{\mathfrak{g}+1}\left(z, n, t_{\mathfrak{g}}\right)+y}{2 a\left(n, t_{\mathfrak{g}}\right) F_{\mathfrak{g}}\left(z, n, t_{\mathfrak{g}}\right)}=\frac{-2 a\left(n, t_{\mathfrak{g}}\right) F_{\mathfrak{g}}\left(z, n+1, t_{\mathfrak{g}}\right)}{G_{\mathfrak{g}+1}\left(z, n, t_{\mathfrak{g}}\right)+y},
$$

using relation (3.43). With the help of $\phi\left(\cdot, n, t_{\mathfrak{g}}\right)$ one defines another meromorphic function $\psi\left(\cdot, n, n_{0}, t_{\mathfrak{g}}, t_{0, \mathfrak{g}}\right)$ on $\mathscr{K}_{\mathfrak{g}} \backslash\left\{P_{\infty_{ \pm}}\right\}$, the Baker-Akhiezer function, by

$$
\begin{aligned}
& \psi\left(P, n, n_{0}, t_{\mathfrak{g}}, t_{0, \mathfrak{g}}\right) \\
&=\exp \left(\int_{t_{0, \mathfrak{g}}}^{t} d s\left(2 a\left(n_{0}, s\right) F_{\mathfrak{g}}\left(z, n_{0}, s\right) \phi\left(P, n_{0}, s\right)+G_{\mathfrak{g}+1}\left(z, n_{0}, s\right)\right)\right) \\
& \times \begin{cases}\prod_{m=n_{0}}^{n-1} \phi\left(P, m, t_{\mathfrak{g}}\right) & \text { for } n \geq n_{0}+1, \\
1 & \text { for } n=n_{0}, \\
\prod_{m=n}^{n_{0}-1} \phi\left(P, m, t_{\mathfrak{g}}\right)^{-1} & \text { for } n \leq n_{0}-1 .\end{cases}
\end{aligned}
$$

The divisor $\left(\phi\left(\cdot, n, t_{\mathfrak{g}}\right)\right)$ of $\phi\left(\cdot, n, t_{\mathfrak{g}}\right)$ is given by

$$
\left(\phi\left(\cdot, n, t_{\mathfrak{g}}\right)\right)=\mathscr{D}_{P_{\infty+} \underline{\hat{\mu}}\left(n+1, t_{\mathfrak{g}}\right)}-\mathscr{D}_{P_{\infty}-\underline{\hat{\mu}}\left(n, t_{\mathfrak{g}}\right)},
$$

where

$$
\underline{\hat{\mu}}\left(m, t_{\mathfrak{g}}\right)=\left(\hat{\mu}_{1}\left(m, t_{\mathfrak{g}}\right), \ldots, \hat{\mu}_{\mathfrak{g}}\left(m, t_{\mathfrak{g}}\right)\right) \in \sigma^{\mathfrak{g}} \mathscr{K}_{\mathfrak{g}}, \quad m \in \mathrm{Z},
$$

$$
\hat{\mu}_{j}\left(n, t_{\mathfrak{g}}\right)=\left(\mu_{j}\left(n, t_{\mathfrak{g}}\right),-G_{\mathfrak{g}+1}\left(\mu_{j}\left(n, t_{\mathfrak{g}}\right), n, t_{\mathfrak{g}}\right)\right), \quad j=1, \ldots, \mathfrak{g},
$$

(3.51c) $\hat{\mu}_{j}\left(n+1, t_{\mathfrak{g}}\right)=\left(\mu_{j}\left(n+1, t_{\mathfrak{g}}\right), G_{\mathfrak{g}+1}\left(\mu_{j}\left(n+1, t_{\mathfrak{g}}\right), n, t_{\mathfrak{g}}\right)\right)$,

$$
j=1, \ldots, \mathfrak{g} .
$$

The sGmKdV hierarchy. The combined sine-Gordon and $\mathrm{mKdV}$ hierarchy is defined in terms of a zero curvature formalism as follows. Introduce the 
DUBROVIN EQUATIONS AND INTEGRABLE SYSTEMS ON HYPERELLIPTIC . . . 103

$2 \times 2$ matrices

$$
U\left(z, x, t_{\mathfrak{g}}\right)=-i\left(\begin{array}{cc}
\frac{1}{2} u_{x}\left(x, t_{\mathfrak{g}}\right) & 1 \\
z & -\frac{1}{2} u_{x}\left(x, t_{\mathfrak{g}}\right)
\end{array}\right), \quad\left(x, t_{\mathfrak{g}}\right) \in \mathrm{R}^{2},
$$

and

$$
V_{\mathfrak{g}}\left(z, x, t_{\mathfrak{g}}\right)=\left(\begin{array}{cc}
-G_{\mathfrak{g}-1}\left(z, x, t_{\mathfrak{g}}\right) & \frac{1}{z} F_{\mathfrak{g}}\left(z, x, t_{\mathfrak{g}}\right) \\
H_{\mathfrak{g}}\left(z, x, t_{\mathfrak{g}}\right) & G_{\mathfrak{g}-1}\left(z, x, t_{\mathfrak{g}}\right)
\end{array}\right), \quad \begin{aligned}
& \left(x, t_{\mathfrak{g}}\right) \in \mathrm{R}^{2}, \\
& \mathfrak{g} \in \mathrm{N}_{0}
\end{aligned}
$$

Then the zero curvature relation reads

$$
U_{t_{\mathfrak{g}}}-V_{\mathfrak{g}, x}+\left[U, V_{\mathfrak{g}}\right]=0, \quad \mathfrak{g} \in \mathrm{N}_{0},
$$

resulting in the equations

$$
u_{x t_{\mathfrak{g}}}\left(x, t_{\mathfrak{g}}\right)=-2 i G_{\mathfrak{g}-1, x}\left(x, t_{\mathfrak{g}}\right)-2\left(H_{\mathfrak{g}}\left(x, t_{\mathfrak{g}}\right)-F_{\mathfrak{g}}\left(x, t_{\mathfrak{g}}\right)\right),
$$

(3.55b) $\quad F_{\mathfrak{g}, x}\left(x, t_{\mathfrak{g}}\right)=-i u_{x}\left(x, t_{\mathfrak{g}}\right) F_{\mathfrak{g}}\left(x, t_{\mathfrak{g}}\right)-2 i z G_{\mathfrak{g}-1}\left(x, t_{\mathfrak{g}}\right)$,

(3.55c) $H_{\mathfrak{g}, x}\left(x, t_{\mathfrak{g}}\right)=i u_{x}\left(x, t_{\mathfrak{g}}\right) H_{\mathfrak{g}}\left(x, t_{\mathfrak{g}}\right)+2 i z G_{\mathfrak{g}-1}\left(x, t_{\mathfrak{g}}\right)$.

Making the following polynomial ansatz

$$
F_{\mathfrak{g}}\left(z, x, t_{\mathfrak{g}}\right)=\sum_{j=0}^{\mathfrak{g}} f_{\mathfrak{g}-j}\left(x, t_{\mathfrak{g}}\right) z^{j}=\prod_{j=1}^{\mathfrak{g}}\left(z-\mu_{j}\left(x, t_{\mathfrak{g}}\right)\right),
$$

$$
H_{\mathfrak{g}}\left(z, x, t_{\mathfrak{g}}\right)=\sum_{j=0}^{\mathfrak{g}} h_{\mathfrak{g}-j}\left(x, t_{\mathfrak{g}}\right) z^{j}=\prod_{j=1}^{\mathfrak{g}}\left(z-v_{j}\left(x, t_{\mathfrak{g}}\right)\right),
$$

(3.56c) $G_{-1}\left(z, x, t_{\mathfrak{g}}\right)=0, \quad G_{\mathfrak{g}-1}\left(z, x, t_{\mathfrak{g}}\right)=\sum_{j=0}^{\mathfrak{g}-1} g_{\mathfrak{g}-1-j}\left(x, t_{\mathfrak{g}}\right) z^{j}$,

one concludes ${ }^{5}\left(f_{j}=f_{j}\left(x, t_{\mathfrak{g}}\right)\right.$, etc. $)$

$f_{\mathfrak{g}}=\alpha e^{-i u}, h_{\mathfrak{g}}=\beta e^{i u}, \quad \alpha, \beta \in \mathrm{C}, \mathfrak{g} \in \mathrm{N}_{0}$.

$f_{0}=1, \quad f_{j, x}=-\frac{1}{4} f_{j-1, x x x}+w_{+} f_{j-1, x}+\frac{1}{2} w_{+, x} f_{j-1}, j=1, \ldots, \mathfrak{g}, \mathfrak{g} \in \mathrm{N}$,

$h_{0}=1, \quad h_{j, x}=-\frac{1}{4} h_{j-1, x x x}+w_{-} h_{j-1, x}+\frac{1}{2} w_{-, x} h_{j-1}, j=1, \ldots, \mathfrak{g}, \mathfrak{g} \in \mathrm{N}$,

\footnotetext{
${ }^{5}$ One observes that the recursions (3.57) are identical with the $\mathrm{KdV}$ recursion replacing $V$ by $w_{ \pm}$
} 
where

$$
w_{ \pm}=-\frac{1}{4}\left(u_{x}^{2} \pm 2 i u_{x x}\right)
$$

and

$g_{-1}=0, \quad g_{j}=\frac{i}{2}\left(f_{j, x}+i u_{x} f_{j}\right)=\frac{i}{2}\left(-h_{j, x}+i u_{x} h_{j}\right), \quad j=0, \ldots, \mathfrak{g}-1, \mathfrak{g} \in \mathrm{N}$

(we refer to [28] for a detailed discussion).

REMARK 3.1. The recursion for the sGmKdV hierarchy is anomalous compared to the other hierarchies studied in this paper. For the KdV, AKNS as well as the $\mathrm{Tl}$ hierarchies the functions $f_{j}$ (and $g_{j}$ and $h_{j}$ where applicable) are defined by the same recursion formula for all $j \in \mathrm{N}$ irrespective of the given genus $g$. However, for the $\mathrm{sG}$ hierarchy $f_{\mathfrak{g}}$ and $h_{\mathfrak{g}}$ are always given by (3.57) for $\mathfrak{g} \in \mathrm{N}_{0}$. This raises a compatibility problem in the recursion formalism. A proof of the solvability of the recursion can be found in [28], Appendix C.

The gth sGmKdV equation is then defined by

$$
\begin{aligned}
\operatorname{sGmKdV}_{\mathfrak{g}}\left(u\left(x, t_{\mathfrak{g}}\right)\right)= & u_{x t_{\mathfrak{g}}}\left(x, t_{\mathfrak{g}}\right)+2 i g_{\mathfrak{g}-1, x}\left(x, t_{\mathfrak{g}}\right) \\
& +2\left(\beta e^{i u\left(x, t_{\mathfrak{g}}\right)}-\alpha e^{-i u\left(x, t_{\mathfrak{g}}\right)}\right)=0, \quad g \in \mathbb{N}_{0} .
\end{aligned}
$$

One observes that $\alpha=\beta=i / 4$ and $g=0$ yields the well-known sineGordon equation in light-cone coordinates. Appropriate choices of $\alpha$ and $\beta$ in (3.60) include the sinh-Gordon hierarchy, the corresponding elliptic equations, the Liouville model, as well as the modified $\mathrm{KdV}$ hierarchy (taking $\alpha=\beta=$ $0)$.

In the stationary case, where $u_{x t_{\mathfrak{g}}}=0$, one finds

$$
\frac{d}{d x}\left(z G_{\mathfrak{g}-1}(z, x)^{2}+F_{\mathfrak{g}}(z, x) H_{\mathfrak{g}}(z, x)\right)=0
$$

and hence

$$
{ }_{z} G_{\mathfrak{g}-1}(z, x)^{2}+F_{\mathfrak{g}}(z, x) H_{\mathfrak{g}}(z, x)=P_{2 \mathfrak{g}}(z),
$$

where $P_{2 \mathfrak{g}}(z)$ is $x$-independent. It is more convenient to define $R_{2 \mathfrak{g}+1}(z)=$ $z P_{2 \mathfrak{g}}(z)$ so that (3.62) becomes

$$
z^{2} G_{\mathfrak{g}-1}(z, x)^{2}+z F_{\mathfrak{g}}(z, x) H_{\mathfrak{g}}(z, x)=R_{2 \mathfrak{g}+1}(z),
$$


where $R_{2 \mathfrak{g}+1}$ is a monic polynomial in $z$ of degree $2 \mathfrak{g}+1$ of the form

$$
R_{2 \mathfrak{g}+1}(z)=\prod_{m=0}^{2 \mathfrak{g}}\left(z-E_{m}\right), \quad E_{0}=0, \quad E_{1}, \ldots, E_{2 \mathfrak{g}} \in \mathrm{C} .
$$

This polynomial defines the hyperelliptic curve $\mathscr{K}_{\mathfrak{g}}$ of genus $\mathfrak{g}$ by the relation $y^{2}-R_{2 \mathfrak{g}+1}(z)=0$ and hence $N=2 \mathfrak{g}$ in the terminology of Section $2 . \mathscr{K}_{\mathfrak{g}}$ is compactified by adding a point $P_{\infty}$.

REMARK 3.2. (i) One observes that the sGmKdV-curve is a special case of the $\mathrm{KdV}$ curve with the additional constraint $E_{0}=0$.

(ii) In the stationary case the choice of $\alpha$ and $\beta$ is constrained by the relation

$$
\alpha \beta=\prod_{j=1}^{2 \mathrm{~g}} E_{j}
$$

as can be seen by inserting $z=0$ in (3.63), using (3.57) and (3.64).

We now return to the time-dependent formalism. Let $\phi\left(\cdot, x, t_{\mathfrak{g}}\right)$ be the meromorphic function on $\mathscr{K}_{\mathfrak{g}}$ given by

$$
\phi\left(P, x, t_{\mathrm{g}}\right)=\frac{y-z G_{\mathrm{g}-1}\left(z, x, t_{\mathrm{g}}\right)}{F_{\mathrm{g}}\left(z, x, t_{\mathrm{g}}\right)}=\frac{z H_{\mathfrak{g}}\left(z, x, t_{\mathrm{g}}\right)}{y+z G_{\mathrm{g}-1}\left(z, x, t_{\mathrm{g}}\right)} .
$$

Hence the divisor $\left(\phi\left(\cdot, x, t_{\mathfrak{g}}\right)\right)$ of $\phi\left(\cdot, x, t_{\mathfrak{g}}\right)$ reads

$$
\left(\phi\left(\cdot, x, t_{\mathfrak{g}}\right)\right)=\mathscr{D}_{Q_{0} \underline{\underline{v}}\left(x, t_{\mathrm{g}}\right)}-\mathscr{D}_{P_{\infty} \underline{\mu}\left(x, t_{\mathrm{g}}\right)},
$$

with $\hat{\mu}\left(x, t_{\mathfrak{g}}\right)=\left(\hat{\mu}_{1}\left(x, t_{\mathfrak{g}}\right), \ldots, \hat{\mu}_{\mathfrak{g}}\left(x, t_{\mathfrak{g}}\right)\right), \underline{\hat{v}}\left(x, t_{\mathfrak{g}}\right)=\left(\hat{v}_{1}\left(x, t_{\mathfrak{g}}\right), \ldots, \hat{v}_{\mathfrak{g}}\left(x, t_{\mathfrak{g}}\right)\right) \in$ $\sigma^{\mathfrak{g}} \overline{\mathscr{K}_{\mathrm{g}}}$,

(3.68a) $\hat{\mu}_{j}\left(x, t_{r}\right)$

$$
=\left(\mu_{j}\left(x, t_{r}\right),-\mu_{j}\left(x, t_{r}\right) G_{\mathfrak{g}-1}\left(\mu_{j}\left(x, t_{r}\right), x, t_{r}\right)\right) \in \mathscr{K}_{\mathfrak{g}}, j=1, \ldots, \mathfrak{g},
$$

(3.68b) $\quad \hat{v}_{j}\left(x, t_{r}\right)$

$$
=\left(v_{j}\left(x, t_{r}\right), v_{j}\left(x, t_{r}\right) G_{\mathfrak{g}-1}\left(v_{j}\left(x, t_{r}\right), x, t_{r}\right)\right) \in \mathscr{K}_{\mathfrak{g}}, j=1, \ldots, \mathfrak{g} .
$$

The time-dependent Baker-Akhiezer vector

$$
\Psi\left(P, x, x_{0}, t_{\mathfrak{g}}, t_{0, \mathfrak{g}}\right)=\left(\begin{array}{l}
\psi_{1}\left(P, x, x_{0}, t_{\mathfrak{g}}, t_{0, \mathfrak{g}}\right) \\
\psi_{2}\left(P, x, x_{0}, t_{\mathfrak{g}}, t_{0, \mathfrak{g}}\right)
\end{array}\right)
$$


is defined on $\mathscr{K}_{\mathfrak{g}} \backslash\left\{P_{\infty}\right\}$ by

(3.70) $\quad \psi_{1}\left(P, x, x_{0}, t_{\mathfrak{g}}, t_{0, \mathfrak{g}}\right)=\exp \left(-\frac{i}{2}\left(u\left(x, t_{\mathfrak{g}}\right)-u\left(x_{0}, t_{\mathfrak{g}}\right)\right)\right.$

$\left.+i \int_{x_{0}}^{x} d x^{\prime} \phi\left(P, x^{\prime}, t_{\mathfrak{g}}\right)-\int_{t_{0, \mathfrak{g}}}^{t_{\mathfrak{g}}} d s\left(z^{-1} F_{\mathfrak{g}}\left(z, x_{0}, s\right) \phi\left(P, x_{0}, s\right)+G_{\mathfrak{g}-1}\left(z, x_{0}, s\right)\right)\right)$,

$$
\psi_{2}\left(P, x, x_{0}, t_{\mathfrak{g}}, t_{0, \mathfrak{g}}\right)=-\psi_{1}\left(P, x, x_{0}, t_{\mathfrak{g}}, t_{0, \mathfrak{g}}\right) \phi\left(P, x, t_{\mathfrak{g}}\right) .
$$

Combining relations (3.56a), (3.56b), and (3.57) one concludes

$$
\begin{aligned}
u\left(x, t_{\mathfrak{g}}\right) & =i \ln \left((-1)^{\mathfrak{g}} \alpha^{-1} \prod_{j=1}^{\mathfrak{g}} \mu_{j}\left(x, t_{\mathfrak{g}}\right)\right) \\
& =-i \ln \left((-1)^{\mathfrak{g}} \beta^{-1} \prod_{j=1}^{\mathfrak{g}} v_{j}\left(x, t_{\mathfrak{g}}\right)\right) .
\end{aligned}
$$

We will also need the following asymptotic high-energy expansion

$$
\frac{F_{\mathrm{g}}\left(z, x, t_{\mathrm{g}}\right)}{R_{2 \mathfrak{g}+1}(z)^{1 / 2}} \underset{z \rightarrow \infty}{=} \frac{1}{z^{1 / 2}} \sum_{j=0}^{\infty} \hat{f_{j}}\left(x, t_{\mathfrak{g}}\right) z^{-j},
$$

with $\hat{f}_{j}$ denoting the homogeneous coefficients $f_{j}$ in (3.57).

\section{Symmetric functions}

Let $g \in \mathrm{N}$ be fixed and define

$$
\mathscr{S}_{k}=\left\{\underline{\ell}=\left(\ell_{1}, \ldots, \ell_{k}\right) \in \mathrm{N}^{k} \mid \ell_{1}<\cdots<\ell_{k} \leq \mathfrak{g}\right\}, \quad k \leq \mathfrak{g},
$$

$$
\mathscr{I}_{k}^{(j)}=\left\{\underline{\ell}=\left(\ell_{1}, \ldots, \ell_{k}\right) \in \mathscr{S}_{k} \mid \ell_{m} \neq j\right\}, \quad k \leq \mathfrak{g}-1, \quad 1 \leq j \leq \mathfrak{g} .
$$

Define

$$
\Psi_{0}(\underline{\mu})=1, \quad \Psi_{k}(\underline{\mu})=(-1)^{k} \sum_{\underline{\ell} \in \mathscr{S}_{k}} \mu_{\ell_{1}} \cdots \mu_{\ell_{k}}, \quad k \leq \mathfrak{g},
$$

$$
\begin{aligned}
& \Phi_{0}^{(j)}\left(\underline{\mu)}=1, \quad \Phi_{k}^{(j)}\left(\underline{\mu)}=(-1)^{k} \sum_{\underline{\ell} \in \mathscr{I}_{k}^{(j)}} \mu_{\ell_{1}} \cdots \mu_{\ell_{k}}, \quad k \leq \mathfrak{g}-1,\right.\right. \\
& \Phi_{\mathfrak{g}}^{(j)}(\underline{\mu})=0, \quad 1 \leq j \leq \mathfrak{g}
\end{aligned}
$$


where $\mu=\left(\mu_{1}, \ldots, \mu_{\mathfrak{g}}\right) \in \mathrm{C}^{\mathfrak{g}}$. One recognizes the simple pattern,

$$
\Psi_{1}(\underline{\mu})=-\sum_{\ell=1}^{\mathfrak{g}} \mu_{\ell}, \quad \Psi_{2}(\underline{\mu})=\sum_{\substack{\ell_{1}, \ell_{2}=1 \\ \ell_{1}<\ell_{2}}}^{\mathfrak{g}} \mu_{\ell_{1}} \mu_{\ell_{2}}, \quad \text { etc. },
$$

$$
\Phi_{1}^{(j)}(\underline{\mu})=-\sum_{\substack{\ell=1 \\ \ell \neq j}}^{\mathfrak{g}} \mu_{\ell}, \quad \Phi_{2}^{(j)}(\underline{\mu})=\sum_{\substack{\ell_{1}, \ell_{2}=1 \\ \ell_{1}, \ell_{2} \neq j \\ \ell_{1}<\ell_{2}}}^{\mathfrak{g}} \mu_{\ell_{1}} \mu_{\ell_{2}}, \text { etc. }
$$

Let $E_{0}, \ldots, E_{N}$ be $N+1$ complex numbers, where $N=2 \mathfrak{g}$ or $N=2 \mathfrak{g}+1$ depending on the underlying hierarchy of soliton equations. For brevity we introduce $\underline{E}=\left(E_{0}, \ldots, E_{N}\right)$, assume $z \in \mathrm{C}$ such that $|z|>\max \left\{\left|E_{0}\right|, \ldots,\left|E_{N}\right|\right\}$, and recall

$$
\left(\prod_{m=0}^{N}\left(1-\frac{E_{m}}{z}\right)\right)^{-1 / 2}=\sum_{k=0}^{\infty} \hat{c}_{k}(\underline{E}) z^{-k}
$$

where

$$
\begin{aligned}
& \hat{c}_{0}(\underline{E})=1, \\
& \hat{c}_{k}(\underline{E})=\sum_{\substack{j_{0}, \ldots, j_{N}=0 \\
j_{0}+\cdots+j_{N}=k}}^{k} \frac{\left(2 j_{0}\right) ! \ldots\left(2 j_{N}\right) !}{2^{2 k}\left(j_{0} !\right)^{2} \ldots\left(j_{N} !\right)^{2}} E_{0}^{j_{0}} \ldots E_{N}^{j_{N}}, \quad k \in \mathrm{N} .
\end{aligned}
$$

The first few terms read

$$
\begin{aligned}
& \hat{c}_{0}(\underline{E})=1, \quad \hat{c}_{1}(\underline{E})=\frac{1}{2} \sum_{j=0}^{N} E_{j}, \\
& \hat{c}_{2}(\underline{E})=\frac{1}{4} \sum_{\substack{j, k=0 \\
j<k}}^{N} E_{j} E_{k}+\frac{3}{8} \sum_{j=0}^{N} E_{j}^{2}, \quad \text { etc. }
\end{aligned}
$$

Similarly,

$$
\left(\prod_{m=0}^{N}\left(1-\frac{E_{m}}{z}\right)\right)^{1 / 2}=\sum_{k=0}^{\infty} c_{k}(\underline{E}) z^{-k}
$$


where

$$
\cdot \sum_{\substack{j_{0}, \ldots, j_{N}=0 \\ j_{0}+\cdots+j_{N}=k}}^{k} \frac{\left(2 j_{0}\right) ! \ldots\left(2 j_{N}\right) !}{2^{2 k}\left(j_{0} !\right)^{2} \ldots\left(j_{N} !\right)^{2}\left(2 j_{0}-1\right) \ldots\left(2 j_{N}-1\right)} E_{0}^{j_{0}} \ldots E_{N}^{j_{N}}, \quad k \in \mathrm{N} .
$$

Explicitly, one obtains for the first few coefficients

$$
\begin{aligned}
& c_{0}(\underline{E})=1, \quad c_{1}(\underline{E})=-\frac{1}{2} \sum_{j=0}^{N} E_{j}, \\
& c_{2}(\underline{E})=\frac{1}{4} \sum_{\substack{j, k=0 \\
j<k}}^{N} E_{j} E_{k}-\frac{1}{8} \sum_{j=0}^{N} E_{j}^{2}, \quad \text { etc. }
\end{aligned}
$$

Next, assuming $\mu_{j} \neq \mu_{j^{\prime}}$ for $j \neq j^{\prime}$, introduce the $\mathfrak{g} \times \mathfrak{g}$ matrix $U_{\mathfrak{g}}(\underline{\mu})$ by

$$
U_{1}(\underline{\mu})=1, \quad U_{\mathfrak{g}}(\underline{\mu})=\left(\frac{\mu_{k}^{j-1}}{\prod_{m \neq k}^{\mathfrak{g}}\left(\mu_{k}-\mu_{m}\right)}\right)_{j, k=1}^{\mathfrak{g}} .
$$

Lemma 4.1. Suppose $\mu_{j} \neq \mu_{j^{\prime}}$ for $j \neq j^{\prime}$. Then

$$
U_{\mathfrak{g}}(\underline{\mu})^{-1}=\left(\Phi_{\mathfrak{g}-k}^{(j)}(\underline{\mu})\right)_{j, k=1}^{\mathfrak{g}} .
$$

Proof. First we observe that we may write

$$
U_{\mathfrak{g}}(\underline{\mu})=\left(\frac{\mu_{k}^{j-1}}{F_{\mathfrak{g}}^{\prime}\left(\mu_{k}\right)}\right)_{j, k=1}^{\mathfrak{g}} .
$$

Using Lagrange's interpolation result, Theorem A.1 (replacing $k$ by $\mathfrak{g}-k$ in (A.1)), proves the result.

Of crucial importance for our approach is the fact that we are able to express $f_{j}$ and $F_{r}$ in terms of elementary symmetric functions of $\mu_{1}, \ldots, \mu_{\mathfrak{g}}$. The expression is given below for the homogeneous case only, denoted by $\hat{f}_{j}$ and $\widehat{F}_{r}$, where the integration constants $c_{\ell}$ for $\ell \in \mathrm{N}$ vanish. We start with $\hat{f}_{j}$. 
Lemma 4.2. Let $\hat{c}_{j}(\underline{E})$ and $c_{j}(\underline{E})$ be defined as in (4.5) and (4.8). Then one infers the following results for the KdV and the Toda hierarchies ${ }^{6}$,

$$
\hat{f_{j}}=\sum_{k=0}^{j \wedge \mathfrak{g}} \hat{c}_{j-k}(\underline{E}) \Psi_{k}(\underline{\mu}), \quad \Psi_{j}(\underline{\mu})=f_{j}=\sum_{k=0}^{j} c_{j-k}(\underline{E}) \hat{f}_{k} .
$$

For the AKNS hierarchy one obtains

$$
\hat{f}_{j}=-i q \sum_{k=0}^{j \wedge \mathfrak{g}} \hat{c}_{j-k}(\underline{E}) \Psi_{k}(\underline{\mu}), \quad \hat{h}_{j}=i p \sum_{k=0}^{j \wedge \mathfrak{g}} \hat{c}_{j-k}(\underline{E}) \Psi_{k}(\underline{\nu}),
$$

$$
-i q \Psi_{j}(\underline{\mu})=f_{j}=\sum_{k=0}^{j} c_{j-k}(\underline{E}) \hat{f}_{k}, \quad i p \Psi_{j}(\underline{v})=h_{j}=\sum_{k=0}^{j} c_{j-k}(\underline{E}) \hat{h}_{k},
$$

where $\underline{v}=\left(v_{1}, \ldots, v_{\mathrm{g}}\right)$

In the sGmKdV case one has

$$
\hat{f}_{j}=\sum_{k=0}^{j \wedge \mathfrak{g}} \hat{c}_{j-k}(\underline{E}) \Psi_{k}(\underline{\mu}), \quad \hat{h}_{j}=\sum_{k=0}^{j \wedge \mathfrak{g}} \hat{c}_{j-k}(\underline{E}) \Psi_{k}(\underline{v}),
$$

$$
\Psi_{j}(\underline{\mu})=f_{j}=\sum_{k=0}^{j} c_{j-k}(\underline{E}) \hat{f}_{k}, \quad \Psi_{j}(\underline{\nu})=h_{j}=\sum_{k=0}^{j} c_{j-k}(\underline{E}) \hat{h}_{k},
$$

and

$$
\hat{f}_{\mathfrak{g}}=\Psi_{\mathfrak{g}}(\underline{\mu})=f_{\mathfrak{g}}, \quad \hat{h}_{\mathfrak{g}}=\Psi_{\mathfrak{g}}(\underline{\nu})=h_{\mathfrak{g}}, \quad \mathfrak{g} \in \mathrm{N}_{0} .
$$

Proof. The proof is identical in all cases, and is based on the high-energy expansion of the Green's function of the corresponding linear operator $L$ or $M$ in the Lax pair or zero curvature formulation of the hierarchy considered. We provide the details for the KdV hierarchy only.

\footnotetext{
${ }^{6} n \wedge m=\min \{n, m\}$.
} 
Using (4.4) one finds

$$
\begin{aligned}
\frac{F_{\mathfrak{g}}(z)}{R_{2 \mathfrak{g}+1}(z)^{1 / 2}} & =\frac{\prod_{j=1}^{\mathfrak{g}}\left(z-\mu_{j}\right)}{R_{2 \mathfrak{g}+1}(z)^{1 / 2}}=\frac{1}{z^{1 / 2}} \frac{\prod_{j=1}^{\mathfrak{g}}\left(1-\frac{\mu_{j}}{z}\right)}{\prod_{m=0}^{2 \mathfrak{g}}\left(1-\frac{E_{m}}{z}\right)^{1 / 2}} \\
& =\frac{1}{z^{1 / 2}}\left(\sum_{j=0}^{\mathfrak{g}} \Psi_{j}(\underline{\mu}) z^{-j}\right)\left(\sum_{m=0}^{\infty} c_{m}(\underline{E}) z^{-m}\right) \\
& =\frac{1}{z^{1 / 2}} \sum_{m=0}^{\infty} z^{-m} \sum_{k=0}^{m \wedge \mathfrak{g}} c_{m-k}(\underline{E}) \Psi_{k}(\underline{\mu}) .
\end{aligned}
$$

Combining this result with the high-energy expansion (3.11) proves (4.13) for $\hat{f}_{j}$. Next, multiplying (4.4) and (4.7), a comparison of coefficients of $z^{-k}$ yields

$$
\sum_{\ell=0}^{k} \hat{c}_{k-\ell}(\underline{E}) c_{\ell}(\underline{E})=\delta_{k, 0}, \quad k \in \mathrm{N}_{0} .
$$

Thus, one computes

$$
\begin{aligned}
\sum_{m=0}^{j} c_{j-m}(\underline{E}) \hat{f}_{m} & =\sum_{m=0}^{j} \sum_{k=0}^{m} c_{j-m}(\underline{E}) \hat{c}_{m-k}(\underline{E}) f_{k} \\
& =\sum_{k=0}^{j} \sum_{p=k}^{j} c_{j-p}(\underline{E}) \hat{c}_{p-k}(\underline{E}) f_{k} \\
& =\sum_{k=0}^{j}\left(\sum_{m=0}^{j-k} c_{j-k-m}(\underline{E}) \hat{c}_{m}(\underline{E})\right) f_{k}=f_{j},
\end{aligned}
$$

applying (4.19). Hence one obtains (4.13) for $f_{j}=\Psi_{j}(\underline{\mu})$ (cf. (3.5) and (4.2a)).

REMARK 4.3. One observes that the right-hand side of (4.13) is defined for all $x \in \mathrm{R}$, but when one samples it at integer values $x=n \in \mathrm{Z}$, it coincides with $\hat{f}_{j}(n)$ for the Toda lattice. Thus we have in some sense a continuous extension of the Toda hierarchy (cf. also Lemma 5.6).

Next we introduce

$$
\widehat{F}_{r}(z)=\sum_{\ell=0}^{r} \hat{f}_{r-\ell} z^{\ell}
$$


in the KdV, Toda, and AKNS cases (and similarly $\widehat{H}_{r}(z)=\sum_{\ell=0}^{r} \hat{h}_{r-\ell} z^{\ell}$ in the AKNS case) and

$$
\widehat{F}_{s}(z)=\sum_{\ell=0}^{s} \hat{f}_{s-\ell} z^{\ell}, \quad s=0, \ldots, r-1, \quad \widehat{F}_{r}(z)=\hat{f}_{r}+z \widehat{F}_{r-1}(z)
$$

with $\hat{f}_{r}=\tilde{\alpha} e^{-i u}$ in the SGmKdV case.

THEOREM 4.4. Let $r \in \mathrm{N}_{0}$. For both the KdV and the Tl case one derives ${ }^{7}$

$$
\widehat{F}_{r}\left(\mu_{j}\right)=\sum_{p=(r-\mathfrak{g}) \vee 0}^{r} c_{p}(\underline{E}) \Phi_{r-p}^{(j)}(\underline{\mu}) .
$$

For the AKNS hierarchy one infers

$$
\widehat{F}_{r}\left(\mu_{j}\right)=-i q \sum_{p=(r-\mathfrak{g}) \vee 0}^{r} c_{p}(\underline{E}) \Phi_{r-p}^{(j)}(\underline{\mu}), \quad \widehat{H}_{r}\left(v_{j}\right)=i p \sum_{p=(r-\mathfrak{g}) \vee 0}^{r} c_{p}(\underline{E}) \Phi_{r-p}^{(j)}(\underline{v}) \text {. }
$$

For the sGmKdV hierarchy one concludes ${ }^{8}$

$$
\begin{aligned}
\frac{\widehat{F}_{r}\left(\mu_{j}\right)}{\mu_{j}} & =\sum_{p=(r-1-\mathfrak{g}) \vee 0}^{r-1} c_{p}(\underline{E}) \Phi_{r-1-p}^{(j)}(\underline{\mu})-\frac{\tilde{\alpha}}{\alpha} \Phi_{\mathfrak{g}-1}^{(j)}(\underline{\mu}), \\
\frac{\widehat{H}_{r}\left(v_{j}\right)}{v_{j}} & =\sum_{p=(r-1-\mathfrak{g}) \vee 0}^{r-1} c_{p}(\underline{E}) \Phi_{r-1-p}^{(j)}(\underline{\nu})-\frac{\tilde{\beta}}{\beta} \Phi_{\mathfrak{g}-1}^{(j)}(\underline{\nu}) .
\end{aligned}
$$

Proof. It suffices to consider the KdV and $\mathrm{sG}$ cases. By definition

$$
\widehat{F}_{r}(z)=\sum_{\ell=0}^{r} \hat{f}_{r-\ell} z^{\ell}=\sum_{\ell=0}^{r} z^{\ell} \sum_{m=0}^{(r-\ell) \wedge \mathfrak{g}} \Psi_{m}(\underline{\mu}) c_{r-\ell-m}(\underline{E}) .
$$

Consider first the case $r \leq g$. Then

$$
\widehat{F}_{r}(z)=\sum_{p=0}^{r} c_{p}\left(\underline{E)} \sum_{\ell=0}^{r-p} z^{\ell} \Psi_{r-\ell-p}(\underline{\mu})\right.
$$

\footnotetext{
${ }^{7} n \vee m=\max \{n, m\}$.

${ }^{8}$ Since $r$ is independent of $\mathfrak{g}$, one obtains $\hat{f}_{r}=\tilde{\alpha} e^{-i u}, \hat{h}_{r}=\tilde{\beta} e^{i u}$ with $\tilde{\alpha}, \tilde{\beta} \in \mathrm{C}$ independent of $\alpha, \beta$, and $\hat{f}_{q}, \hat{h}_{q}, q=1, \ldots, r-1$ constructed according to (3.57) and (3.59).
} 
and hence

$$
\widehat{F}_{r}\left(\mu_{j}\right)=\sum_{p=0}^{r} c_{p}(\underline{E}) \Phi_{r-p}^{(j)}(\underline{\mu}),
$$

using (A.10). In the case when $r \geq \mathfrak{g}+1$ one finds

$$
\begin{aligned}
\widehat{F}_{r}(z) & =\sum_{m=0}^{\mathfrak{g}} \Psi_{m}(\underline{\mu}) \sum_{p=0}^{r-m} z^{r-m-p} c_{p}(\underline{E}) \\
& \left.=\sum_{p=0}^{r-\mathfrak{g}} c_{p}(\underline{E})\left(\sum_{\ell=0}^{\mathfrak{g}} \Psi_{\ell} \underline{(\mu}\right) z^{\mathfrak{g}-\ell}\right) z^{r-\mathfrak{g}-p}+\sum_{p=r-\mathfrak{g}+1}^{r} c_{p}(\underline{E}) \sum_{\ell=0}^{r-p} \Psi_{\ell}(\underline{\mu}) z^{r-p-\ell} \\
& =F_{\mathfrak{g}}(z) \sum_{p=0}^{r-\mathfrak{g}} c_{p}(\underline{E}) z^{r-\mathfrak{g}-p}+\sum_{p=r-\mathfrak{g}+1}^{r} c_{p}(\underline{E}) \sum_{\ell=0}^{r-p} \Psi_{\ell}(\underline{\mu}) z^{r-p-\ell} \\
& =F_{\mathfrak{g}}(z) \sum_{p=0}^{r-\mathfrak{g}} c_{p}(\underline{E}) z^{r-\mathfrak{g}-p}+\sum_{p=r-\mathfrak{g}+1}^{r} c_{p}(\underline{E}) \sum_{m=0}^{r-p} \Psi_{r-p-m}(\underline{\mu}) z^{m} .
\end{aligned}
$$

Hence,

$$
\widehat{F}_{r}\left(\mu_{j}\right)=\sum_{p=r-\mathfrak{g}+1}^{r} c_{p}(\underline{E}) \Phi_{r-p}^{(j)}(\underline{\mu}),
$$

by using (A.10) again.

In the sGmKdV case one first observes that $\widehat{F}_{r}(z)=z \widehat{F}_{r-1}(z)+\hat{f}_{r}$ implies (4.32)

$$
\frac{\widehat{F}_{r}\left(\mu_{j}\right)}{\mu_{j}}=\widehat{F}_{r-1}\left(\mu_{j}\right)+\frac{\hat{f}_{r}}{\mu_{j}}=\sum_{p=(r-1-\mathfrak{g}) \vee 0}^{r-1} c_{p}(\underline{E}) \Phi_{r-1-p}^{(j)}(\underline{\mu})-\frac{\tilde{\alpha}}{\alpha} \Phi_{\mathfrak{g}-1}^{(j)}(\underline{\mu}),
$$

using $\hat{f}_{r}=\tilde{\alpha} e^{-i u}$ and the trace relation (3.72).

\section{Dubrovin equations and linearized flows}

Dubrovin [21] made the fundamental observation that the Dirichlet divisors for the KdV equation satisfy a first-order system of differential equations. Solving this system can then be used to recover the function $V$ by appealing to a trace formula (cf. (5.22)).

Before stating the Dubrovin equations we need some notation. Let $g \in N$. We start by constructing the hierarchies as explained in Section 3. In particular, we construct the polynomial $F_{\mathfrak{g}}$ with its zeros $\mu=\left(\mu_{1}, \ldots, \mu_{\mathfrak{g}}\right)$, and define the 
corresponding hyperelliptic curve $\mathscr{K}_{\mathrm{g}}$. (In the AKNS case one also constructs the polynomial $H_{\mathrm{g}}$.) Next, fix an $r \in \mathrm{N}_{0}$, and construct the polynomial $F_{r}$. The integration constants in the definition of $F_{r}$ are assumed to be independent of those used to construct $F_{\mathrm{g}}$, and to emphasize this fact we denote it by $\widetilde{F}_{r}$ and the corresponding constants by $\tilde{c}_{\ell}$. The Dubrovin equations give the evolution of $\mu=\left(\mu_{1}, \ldots, \mu_{\mathfrak{g}}\right)$ in terms of the deformation (time) parameter $t_{r}$ according to the $r$ th equation in the hierarchy considered.

The KdV hierarchy. In our setting the Dubrovin equations for the $\mathrm{KdV}$ hierarchy read [8], Sect. 3.5, [16], [18], Sect. 12.3, [27], [31], [34], Chs. 10, 12, [35], Ch. 4,

$$
\begin{aligned}
\frac{\partial}{\partial x} \mu_{j}\left(x, t_{r}\right) & =-2 i \frac{y\left(\hat{\mu}_{j}\left(x, t_{r}\right)\right)}{\prod_{\ell \neq j}^{g}\left(\mu_{j}\left(x, t_{r}\right)-\mu_{\ell}\left(x, t_{r}\right)\right)} \\
\frac{\partial}{\partial t_{r}} \mu_{j}\left(x, t_{r}\right) & =\widetilde{F}_{r}\left(\mu_{j}\left(x, t_{r}\right), x, t_{r}\right) \frac{\partial}{\partial x} \mu_{j}\left(x, t_{r}\right) \\
& =-2 i \frac{y\left(\hat{\mu}_{j}\left(x, t_{r}\right)\right)}{\prod_{\ell \neq j}^{g}\left(\mu_{j}\left(x, t_{r}\right)-\mu_{\ell}\left(x, t_{r}\right)\right)} \widetilde{F}_{r}\left(\mu_{j}\left(x, t_{r}\right), x, t_{r}\right)
\end{aligned}
$$

for $j=1, \ldots, \mathfrak{g}$, with initial data on $\mathscr{K}_{\mathfrak{g}}$ equal to $\underline{\hat{\mu}}\left(\cdot, t_{0, r}\right)=\underline{\hat{\mu}}^{(0)}(\cdot)$, where $\hat{\mu}=\left(\hat{\mu}_{1}, \ldots, \hat{\mu}_{\mathrm{g}}\right)$ and

$$
\hat{\mu}_{j}\left(x, t_{r}\right)=\left(\mu_{j}\left(x, t_{r}\right),-\frac{i}{2} F_{\mathfrak{g}, x}\left(\mu_{j}\left(x, t_{r}\right), x, t_{r}\right)\right) \in \mathscr{K}_{\mathfrak{g}}, \quad j=1, \ldots, \mathfrak{g} .
$$

We remark that (5.1a) is an immediate consequence of (3.5) and (3.15), while (5.1b) follows from (3.15) and

$$
F_{\mathfrak{g}, t_{r}}=\widetilde{F}_{r} F_{\mathfrak{g}, x}-\widetilde{F}_{r, x} F_{\mathfrak{g}}
$$

upon taking $z=\mu_{j}\left(x, t_{r}\right)$.

The AKNS hierarchy. In this case the Dubrovin equations for $\hat{\mu}=\left(\hat{\mu}_{1}, \ldots\right.$, $\left.\hat{\mu}_{\mathfrak{g}}\right)$ are given by [15], [30],

$$
\frac{\partial}{\partial x} \mu_{j}\left(x, t_{r}\right)=-2 i \frac{y\left(\hat{\mu}_{j}\left(x, t_{r}\right)\right)}{\prod_{\ell \neq j}^{\mathfrak{g}}\left(\mu_{j}\left(x, t_{r}\right)-\mu_{\ell}\left(x, t_{r}\right)\right)},
$$

$$
\begin{aligned}
\frac{\partial}{\partial t_{r}} \mu_{j}\left(x, t_{r}\right) & =-\frac{\widetilde{F}_{r}\left(\mu_{j}\left(x, t_{r}\right), x, t_{r}\right)}{i q\left(x, t_{r}\right)} \frac{\partial}{\partial x} \mu_{j}\left(x, t_{r}\right) \\
& =2 \frac{y\left(\hat{\mu}_{j}\left(x, t_{r}\right)\right)}{q\left(x, t_{r}\right) \prod_{\ell \neq j}^{\mathfrak{g}}\left(\mu_{j}\left(x, t_{r}\right)-\mu_{\ell}\left(x, t_{r}\right)\right)} \widetilde{F}_{r}\left(\mu_{j}\left(x, t_{r}\right), x, t_{r}\right)
\end{aligned}
$$


for $j=1, \ldots, \mathfrak{g}$, with initial data on $\mathscr{K}_{\mathfrak{g}}$ equal to $\underline{\hat{\mu}}\left(\cdot, t_{0, r}\right)=\underline{\hat{\mu}}^{(0)}(\cdot)$, where

$$
\hat{\mu}_{j}\left(x, t_{r}\right)=\left(\mu_{j}\left(x, t_{r}\right), G_{\mathfrak{g}+1}\left(\mu_{j}\left(x, t_{r}\right), x, t_{r}\right)\right) \in \mathscr{K}_{\mathfrak{g}}, \quad j=1, \ldots, \mathfrak{g} .
$$

For the corresponding evolution of $\underline{\hat{v}}=\left(\hat{v}_{1}, \ldots, \hat{v}_{\mathfrak{g}}\right)$ one has

$$
\frac{\partial}{\partial x} v_{j}\left(x, t_{r}\right)=-2 i \frac{y\left(\hat{v}_{j}\left(x, t_{r}\right)\right)}{\prod_{\ell \neq j}^{\mathfrak{g}}\left(v_{j}\left(x, t_{r}\right)-v_{\ell}\left(x, t_{r}\right)\right)},
$$

$$
\begin{aligned}
\frac{\partial}{\partial t_{r}} v_{j}\left(x, t_{r}\right) & =\widetilde{H}_{r}\left(v_{j}\left(x, t_{r}\right), x, t_{r}\right) \frac{\partial}{\partial x} v_{j}\left(x, t_{r}\right) \\
& =-2 \frac{y\left(\hat{v}_{j}\left(x, t_{r}\right)\right)}{p\left(x, t_{r}\right) \prod_{\ell \neq j}^{g}\left(v_{j}\left(x, t_{r}\right)-v_{\ell}\left(x, t_{r}\right)\right)} \widetilde{H}_{r}\left(v_{j}\left(x, t_{r}\right), x, t_{r}\right)
\end{aligned}
$$

for $j=1, \ldots, \mathfrak{g}$, with initial data on $\mathscr{K}_{\mathfrak{g}}$ equal to $\underline{\hat{v}}\left(\cdot, t_{0, r}\right)=\underline{\hat{v}}^{(0)}(\cdot)$, where

$$
\hat{v}_{j}\left(x, t_{r}\right)=\left(v_{j}\left(x, t_{r}\right),-G_{\mathfrak{g}+1}\left(v_{j}\left(x, t_{r}\right), x, t_{r}\right)\right) \in \mathscr{K}_{\mathfrak{g}}, \quad j=1, \ldots, \mathfrak{g} .
$$

The Toda hierarchy. Here the Dubrovin equations for $\underline{\hat{\mu}}=\left(\hat{\mu}_{1}, \ldots, \hat{\mu}_{\mathfrak{g}}\right)$ read [11], [17], [37], [41], Ch.4,

$$
\frac{\partial}{\partial t_{r}} \mu_{j}\left(n, t_{r}\right)=2 \frac{y\left(\hat{\mu}_{j}\left(n, t_{r}\right)\right)}{\prod_{\ell \neq j}^{\mathfrak{g}}\left(\mu_{j}\left(n, t_{r}\right)-\mu_{\ell}\left(n, t_{r}\right)\right)} \widetilde{F}_{r}\left(\mu_{j}\left(n, t_{r}\right), n, t_{r}\right)
$$

for $j=1, \ldots, \mathfrak{g}$, with initial data on $\mathscr{K}_{\mathfrak{g}}$ equal to $\underline{\hat{\mu}}\left(\cdot, t_{0, r}\right)=\underline{\hat{\mu}}^{(0)}(\cdot)$, where (5.9)

$$
\hat{\mu}_{j}\left(n, t_{r}\right)=\left(\mu_{j}\left(n, t_{r}\right),-G_{\mathfrak{g}+1}\left(\mu_{j}\left(n, t_{r}\right), n, t_{r}\right)\right) \in \mathscr{K}_{\mathfrak{g}}, \quad j=1, \ldots, \mathfrak{g} .
$$

We note that (5.4a) and (5.6a) formally coincide with (5.1a). The case of the Toda hierarchy, however, is quite different since (5.8) concerns the $t_{r}$-dependence of $\hat{\mu}\left(n, t_{r}\right)$ and no analogous first-order nonlinear difference equation concerning the $n$-dependence of $\hat{\mu}\left(n, t_{r}\right)$ (i.e., an analog of (5.1a) or (5.4a)) appears to be known. In this context we refer the reader to Lemma 5.6, where we continue this discussion.

The sGmKdV hierarchy. Finally, in the case of the sGmKdV hierarchy the equations for $\mu$ read [28]

$$
\mu_{j, x}\left(x, t_{r}\right)=-2 i \frac{y\left(\hat{\mu}_{j}\left(x, t_{r}\right)\right)}{\prod_{\substack{\ell=1 \\ \ell \neq j}}^{\mathfrak{g}}\left(\mu_{j}\left(x, t_{r}\right)-\mu_{\ell}\left(x, t_{r}\right)\right)},
$$




$$
\mu_{j, t_{r}}\left(x, t_{r}\right)=2 \frac{\widetilde{F}_{r}\left(\mu_{j}\left(x, t_{r}\right), x, t_{r}\right)}{\mu_{j}\left(x, t_{r}\right)} \frac{y\left(\hat{\mu}_{j}\left(x, t_{r}\right)\right)}{\prod_{\substack{\mathfrak{g}=1 \\ \ell \neq j}}^{\mathfrak{g}}\left(\mu_{j}\left(x, t_{r}\right)-\mu_{\ell}\left(x, t_{r}\right)\right)}
$$

for $j=1, \ldots, \mathfrak{g}$, with initial data on $\mathscr{K}_{\mathfrak{g}}$ equal to $\underline{\hat{\mu}}\left(\cdot, t_{0, r}\right)=\underline{\hat{\mu}}^{(0)}(\cdot)$, where

$\hat{\mu}_{j}\left(x, t_{r}\right)=\left(\mu_{j}\left(x, t_{r}\right),-\mu_{j}\left(x, t_{r}\right) G_{\mathfrak{g}-1}\left(\mu_{j}\left(x, t_{r}\right), x, t_{r}\right)\right) \in \mathscr{K}_{\mathfrak{g}}, \quad j=1, \ldots, \mathfrak{g}$.

The corresponding equations for $\underline{v}$ equal

$$
\begin{aligned}
& \text { (5.12a) } v_{j, x}\left(x, t_{r}\right)=-2 i \frac{y\left(\hat{v}_{j}\left(x, t_{r}\right)\right)}{\prod_{\substack{\ell=1 \\
\ell \neq j}}^{\mathfrak{g}}\left(v_{j}\left(x, t_{r}\right)-v_{\ell}\left(x, t_{r}\right)\right)}, \\
& \text { (5.12b) } v_{j, t_{r}}\left(x, t_{r}\right)=2 \frac{\widetilde{H}_{r}\left(v_{j}\left(x, t_{r}\right), x, t_{r}\right)}{v_{j}\left(x, t_{r}\right)} \frac{y\left(\hat{v}_{j}\left(x, t_{r}\right)\right)}{\prod_{\substack{\ell=1 \\
\ell \neq j}}^{\mathfrak{g}}\left(v_{j}\left(x, t_{r}\right)-v_{\ell}\left(x, t_{r}\right)\right)}
\end{aligned}
$$

for $j=1, \ldots, \mathfrak{g}$, with initial data on $\mathscr{K}_{\mathfrak{g}}$ equal to $\underline{\hat{v}}\left(\cdot, t_{0, r}\right)=\underline{\hat{v}}^{(0)}(\cdot)$, where

$$
\hat{v}_{j}\left(x, t_{r}\right)=\left(v_{j}\left(x, t_{r}\right), v_{j}\left(x, t_{r}\right) G_{\mathfrak{g}-1}\left(v_{j}\left(x, t_{r}\right), x, t_{r}\right)\right) \in \mathscr{K}_{\mathfrak{g}}, \quad j=1, \ldots, \mathfrak{g} .
$$

Next we will prove that the Abel map provides a clever change of coordinates that linearizes the Dubrovin flows. This will turn out to be a consequence of the fact that $\widetilde{F}_{r}\left(\mu_{j}\right)$ can be expressed as a linear combination of the functions $\Phi_{k}^{(j)}$. Using Theorem 4.4 it is immediate that this is not only the case for the $\mathrm{KdV}$ hierarchy, but also for all the other hierarchies discussed in this paper.

THEOREm 5.1. Suppose $\mu=\left(\mu_{1}, \ldots, \mu_{\mathfrak{g}}\right)$ satisfies the Dubrovin equations (5.1) and assume that $\mu_{j} \neq \mu_{j^{\prime}}$ for $j \neq j^{\prime}$. Let $r \in \mathrm{N}_{0}$ and introduce

$$
\widetilde{F}_{r}\left(\mu_{j}\right)=\sum_{k=0}^{r \wedge \mathfrak{g}} d_{r, k} \Phi_{k}^{(j)}(\underline{\mu}), \quad d_{r, 0}, \ldots, d_{r, r \wedge \mathfrak{g}} \in \mathrm{C} .
$$

Then the Abel map

$$
\underline{A}_{P_{0}}\left(\hat{\mu}_{j}\left(x, t_{r}\right)\right)=\left(\underline{A}_{P_{0}, 1}\left(\hat{\mu}_{j}\left(x, t_{r}\right)\right), \ldots, \underline{A}_{P_{0}, \mathrm{~g}}\left(\hat{\mu}_{j}\left(x, t_{r}\right)\right)\right)
$$

linearizes the Dubrovin flows (5.1) in the sense that

$$
\frac{\partial}{\partial t_{r}} \sum_{j=1}^{\mathfrak{g}} \underline{A}_{P_{0}, k}\left(\hat{\mu}_{j}\left(x, t_{r}\right)\right)=-2 i \sum_{\ell=1 \vee(\mathfrak{g}-r)}^{\mathfrak{g}} c_{k, \ell} d_{r, \mathfrak{g}-\ell}
$$


and hence $e^{9}$

$$
\begin{aligned}
\underline{\alpha}_{P_{0}}\left(\mathscr{D}_{\hat{\mu}\left(x, t_{r}\right)}\right)=\underline{\alpha}_{P_{0}}\left(\mathscr{D}_{\hat{\mu}}\left(x_{0}, t_{0, r}\right)\right) \\
\quad-2 i\left(x-x_{0}\right) c_{k, \mathfrak{g}}-2 i\left(t_{r}-t_{0, r}\right) \sum_{\ell=1 \vee(\mathfrak{g}-r)}^{\mathfrak{g}} c_{k, \ell} d_{r, \mathfrak{g}-\ell} .
\end{aligned}
$$

Proof. One computes,

$$
\begin{aligned}
\frac{\partial}{\partial t_{r}} \sum_{j=1}^{\mathfrak{g}} \underline{A}_{P_{0}, k}\left(\hat{\mu}_{j}\left(x, t_{r}\right)\right) & =\frac{\partial}{\partial t_{r}} \sum_{j=1}^{\mathfrak{g}} \int_{P_{0}}^{\hat{\mu}_{j}\left(x, t_{r}\right)} d \omega_{k} \\
& =\frac{\partial}{\partial t_{r}} \sum_{j=1}^{\mathfrak{g}} \sum_{\ell=1}^{\mathfrak{g}} c_{k, \ell} \int_{P_{0}}^{\hat{\mu}_{j}\left(x, t_{r}\right)} \frac{z^{\ell-1} d z}{y(P)} \\
& =\sum_{j=1}^{\mathfrak{g}} \sum_{\ell=1}^{\mathfrak{g}} c_{k, \ell} \frac{\mu_{j}\left(x, t_{r}\right)^{\ell-1}}{y\left(\hat{\mu}_{j}\left(x, t_{r}\right)\right)} \frac{\partial}{\partial t_{r}} \mu_{j}\left(x, t_{r}\right) \\
& =-2 i \sum_{j=1}^{\mathfrak{g}} \sum_{\ell=1}^{\mathfrak{g}} c_{k, \ell} \frac{\mu_{j}\left(x, t_{r}\right)^{\ell-1}}{y\left(\hat{\mu}_{j}\left(x, t_{r}\right)\right)} \\
& \cdot \frac{\prod^{\mathfrak{g}}\left(\hat{\mu}_{j}\left(x, t_{r}\right)\right)}{\left.\prod_{m \neq j}\left(x, t_{r}\right)-\mu_{m}\left(x, t_{r}\right)\right)} \widetilde{F}_{r}\left(\mu_{j}\left(x, t_{r}\right)\right) \\
& =-2 i \sum_{j=1}^{\mathfrak{g}} \sum_{\ell=1}^{\mathfrak{g}} c_{k, \ell} U_{\mathfrak{g}}\left(\mu \underline{\left.\left(x, t_{r}\right)\right)_{\ell, j}} \widetilde{F}_{r}\left(\mu_{j}\left(x, t_{r}\right)\right)\right. \\
& =-2 i \sum_{\ell=1 \vee(\mathfrak{g}-r)}^{\mathfrak{g}} c_{k, \ell} d_{r, \mathfrak{g}-\ell},
\end{aligned}
$$

using Lemma 4.1 in the final step. As for the $x$-variation, one observes that the $t_{0}$-derivative of $\mu_{j}$ coincides with the $x$-derivative in (5.1), and hence it is a special case of (5.18) with $\widetilde{F}_{0}=1$. This proves the theorem.

Corollary 5.2. The Abel map linearizes the Dubrovin flows for the $K d V$, $A K N S, T l$, as well as the $s G K d V$ hierarchies.

\footnotetext{
${ }^{9}$ The situation here resembles the one in classical mechanics where, by a canonical change to cyclic coordinates, the momentum $p_{j}$ becomes a constant of motion and thus $q_{j}(t)=q_{j}\left(t_{0}\right)+$ $p_{j}\left(t-t_{0}\right)$ is linear in time.
} 
Proof. Theorem 4.4 shows that $\widetilde{F}_{r}\left(\mu_{j}\right)$ (and $\widetilde{H}_{r}\left(v_{j}\right)$ in the AKNS case) indeed satisfies the assumption (5.14) of Theorem 5.1, and hence the key calculation (5.18) carries over to the AKNS, Tl, and sGmKdV systems. The special case $r=0$ gives the $x$-variation in all but the $\mathrm{sGmKdV}$ case which, however can easily be verified by explicit computation.

REMARK 5.3. We provide a few more details in the AKNS case. Suppose $\mu$ satisfies (5.4) and similarly, $\underline{v}$ satifies (5.6), with $\mu_{j} \neq \mu_{j^{\prime}}$ and $v_{j} \neq v_{j^{\prime}}$ for $j \neq j^{\prime}$. Let $r \in \mathrm{N}_{0}$ and introduce

$$
\widetilde{F}_{r}\left(\mu_{j}\right)=-i q \sum_{k=0}^{r \wedge \mathfrak{g}} d_{r, k} \Phi_{k}^{(j)} \underline{(\mu)}, \quad \widetilde{H}_{r}\left(v_{j}\right)=i p \sum_{k=0}^{r \wedge \mathfrak{g}} e_{r, k} \Phi_{k}^{(j)}(\underline{v}) .
$$

Then (5.16) and (5.17) hold. In addition, one obtains the following results for the analog of Neumann divisors $\underline{v}\left(x, t_{r}\right)$.

$$
\frac{\partial}{\partial t_{r}} \sum_{j=1}^{\mathfrak{g}} \underline{A}_{P_{0}, k}\left(\hat{v}_{j}\left(x, t_{r}\right)\right)=-2 i \sum_{\ell=1 \vee(\mathfrak{g}-r)}^{\mathfrak{g}} c_{k, \ell} e_{r, \mathfrak{g}-\ell}
$$

and hence

$$
\begin{aligned}
\underline{\alpha}_{P_{0}}\left(\mathscr{D}_{\hat{\hat{v}}\left(x, t_{r}\right)}\right) & =\underline{\alpha}_{P_{0}}\left(\mathscr{D}_{\hat{\hat{v}}\left(x_{0}, t_{0, r}\right)}\right) \\
& -2 i\left(x-x_{0}\right) c_{k, \mathfrak{g}} e_{0,0}-2 i\left(t_{r}-t_{0, r}\right) \sum_{\ell=1 \vee(\mathfrak{g}-r)}^{\mathfrak{g}} c_{k, \ell} e_{r, \mathfrak{g}-\ell} .
\end{aligned}
$$

Necessary and sufficient conditions on Lax pairs to linearize the flow $t \rightarrow$ $L_{t}$ on $J(C)$, where $\left\{L_{t}\right\}$ represents a dymamical system on the Jacobi variety $J(C)$, with $C$ the underlying spectral curve, have been considered by Griffiths [32]. While he considers Lax equations within a cohomological framework, our approach is much more modest in scope but in turn reduces the linearization problem to an elementary exercise in symmetric functions.

Solving these equations one can recover the solution of the integrable equation using trace formulas. For the $\mathrm{KdV}$ hierarchy one has the classical trace formula

$$
V\left(x, t_{r}\right)=\sum_{m=0}^{2 \mathfrak{g}} E_{m}-2 \sum_{j=1}^{\mathfrak{g}} \mu_{j}\left(x, t_{r}\right) .
$$

For the AKNS hierarchy one has

$$
\frac{p_{x}\left(x, t_{r}\right)}{p\left(x, t_{r}\right)}=i \sum_{m=0}^{2 \mathfrak{g}+1} E_{m}-2 i \sum_{j=1}^{\mathfrak{g}} v_{j}\left(x, t_{r}\right),
$$




$$
\frac{q_{x}\left(x, t_{r}\right)}{q\left(x, t_{r}\right)}=-i \sum_{m=0}^{2 g+1} E_{m}+2 i \sum_{j=1}^{g} \mu_{j}\left(x, t_{r}\right)
$$

while for the Toda hierarchy one obtains

$$
\begin{gathered}
a\left(n, t_{r}\right)^{2}=-\frac{1}{2} \sum_{j=1}^{\mathfrak{g}} \frac{G_{\mathfrak{g}+1}\left(\mu_{j}\left(n, t_{r}\right), n, t_{r}\right)}{\prod_{k \neq j}^{\mathfrak{g}}\left(\mu_{j}\left(n, t_{r}\right)-\mu_{k}\left(n, t_{r}\right)\right)}-\frac{1}{4} b\left(n, t_{r}\right)^{2} \\
-\frac{1}{4} \sum_{j=1}^{\mathfrak{g}} \mu_{j}\left(n, t_{r}\right)^{2}+\frac{1}{8} \sum_{m=0}^{2 \mathfrak{g}+1} E_{m}^{2}, \\
b\left(n, t_{r}\right)=-\frac{1}{2} \sum_{m=0}^{2 \mathfrak{g}+1} E_{m}+\sum_{j=1}^{\mathfrak{g}} \mu_{j}\left(n, t_{r}\right) .
\end{gathered}
$$

The "trace" relation for the sGmKdV hierarchy (it would be more appropriate to call this a "determinant" relation) was given in (3.72),

$$
u\left(x, t_{\mathfrak{g}}\right)=i \ln \left((-1)^{\mathfrak{g}} \alpha^{-1} \prod_{j=1}^{\mathfrak{g}} \mu_{j}\left(x, t_{\mathfrak{g}}\right)\right)=-i \ln \left((-1)^{\mathfrak{g}} \beta^{-1} \prod_{j=1}^{\mathfrak{g}} v_{j}\left(x, t_{\mathfrak{g}}\right)\right) .
$$

Remark 5.4. It is important to observe that if one postulates the Dubrovin equations (5.1), and defines $V$ using the trace formula (5.22), one could show by a long and tedious calculation that $V$ indeed satisfies the $r$ th $\mathrm{KdV}$ equation with the correct initial condition. The same applies, of course, to the AKNS, Toda, and sGmKdV hierarchies.

Remark 5.5. For simplicity we assumed $\mu_{j}\left(x, t_{r}\right) \neq \mu_{j^{\prime}}\left(x, t_{r}\right)$ for $j \neq j^{\prime}$ in Theorem 5.1. In the self-adjoint cases, where $\left\{E_{m}\right\}_{m=0, \ldots, N} \subset \mathrm{R}$, this condition is automatically fulfilled for all $\left(x, t_{r}\right) \in \mathrm{R}^{2}$ since then all $\mu_{j}\left(x, t_{r}\right)$ are separated from each other by spectral gaps of $L\left(t_{r}\right)$ or $M\left(t_{r}\right)$. In the general nonself-adjoint case this is no longer true and collisions between the $\mu_{j}$ 's become possible. Nevertheless the Dubrovin equations, properly desingularized near such collision points, stay meaningful as demonstrated in detail by Birnir [9], [10] in the case of complex-valued KdV solutions. In particular, (5.17) (and (5.21)) remain valid in the presence of such collisions due to the continuity of $\underline{\alpha}_{P_{0}}(\cdot)$.

We already mentioned in the paragraph following (5.9) that the Dubrovin equations for the Toda hierarchy differ from the ones associated with the remaining soliton hierarchies in the sense that they do not seem to govern the 
$n$-dependence of $\hat{\mu}\left(n, t_{r}\right)$. We now show how to use Theorem 5.1 to obtain a first-order Dubrovin system for $\hat{\mu}\left(x, t_{r}\right)$ in $x$, whose solution coincides with $\underline{\hat{\mu}}\left(n, t_{r}\right)$ at the integer points $x=n \in Z$. Since the $t_{r}$-dependence of $\underline{\hat{\mu}}$ plays $\bar{n}$ role for this argument, we ignore this dependence in the following result.

Lemma 5.6. Abbreviate $\underline{A}=\left(A_{1}, \ldots, A_{\mathfrak{g}}\right)=\underline{A}_{P_{\infty_{-}}}\left(P_{\infty_{+}}\right)$and $\underline{\hat{\mu}}\left(n_{0}\right)=$ $\hat{\mu}^{(0)}$, with $\hat{\mu}$ defined in (3.51b). Consider the Dubrovin-type system (5.26a)

$\frac{\partial}{\partial x} \mu_{j}(x)=\frac{2 y\left(\hat{\mu}_{j}(x)\right)}{\prod_{\ell \neq j}^{\mathfrak{g}}\left(\mu_{j}(x)-\mu_{\ell}(x)\right)} \sum_{m=1}^{\mathfrak{g}} \Phi_{\mathfrak{g}-m}^{(j)}(\underline{\mu}(x)) \sum_{n=1}^{\mathfrak{g}} c_{m, n}^{-1} A_{n}, \quad j=1, \ldots, \mathfrak{g}$,

$$
\underline{\hat{\mu}}\left(n_{0}\right)=\underline{\hat{\mu}}^{(0)},
$$

with $c_{m, n}$ defined in (2.6). Denote the solution of (5.26) by $\underline{\hat{\mu}}_{0}$. Then $\underline{\hat{\mu}}$ coincides with $\underline{\hat{\mu}}_{0}$ at integer values, that is,

$$
\underline{\hat{\mu}}(n)=\underline{\hat{\mu}}_{0}(n), \quad n \in Z .
$$

Proof. First one recalls the well-known result (see, e.g., [11], Sect. 3)

$$
\underline{\alpha}_{P_{0}}(\mathscr{D} \underline{\hat{\mu}(n)})-\underline{\alpha}_{P_{0}}\left(\mathscr{D}_{\hat{\mu}\left(n_{0}\right)}\right)=\left(n-n_{0}\right) \underline{A}_{P_{\infty_{-}}}\left(P_{\infty_{+}}\right)=\left(n-n_{0}\right) \underline{A} .
$$

In order to complete the proof one only needs to establish that $\underline{\hat{\mu}}_{0}$ satisfies

$$
\underline{\alpha}_{P_{0}}\left(\mathscr{D}_{\hat{\mu}_{0}(x)}\right)-\underline{\alpha}_{P_{0}}\left(\mathscr{D}_{\hat{\mu}_{0}}\left(x_{0}\right)\right)=\left(x-x_{0}\right) \underline{A}_{P_{\infty_{-}}}\left(P_{\infty_{+}}\right)=\left(x-x_{0}\right) \underline{A},
$$

that is, one needs to show

$$
\frac{\partial}{\partial x} \underline{\alpha}_{P_{0}}\left(\mathscr{D}_{\hat{\mu}_{0}(x)}\right)=\underline{A} .
$$

But equation (5.30) follows immediately from (5.8) (identifying $t_{r}$ with $x$ and ignoring its $n$-dependence), (5.14), (5.18) (multiplied by $i$ ), and (5.26).

Thus, the solution $\underline{\hat{\mu}}_{0}(x)$ of (5.26) provides a continuous interpolation for $\hat{\mu}(n)$. In fact, it was our attempt to prove a result like Lemma 5.6 which led us to reconsider Dubrovin equations and ultimately resulted in Theorem 4.4, the explicit connection between $\Phi_{k}^{(j)}(\underline{\mu})$ and the polynomials $\widehat{F}_{r}(z, x)$ defining the hierarchy in question. A first-order system of difference equations for $\mu_{j}$ was recently derived in [43].

Toda systems as integrable discretizations of continuous systems are also studied in [3]. 


\section{Examples}

$K d V$ and $s G$. Pick $E_{0}=0$ and $E_{1}, \ldots, E_{2 \mathfrak{g}} \in \mathrm{C}, E_{m} \neq E_{m^{\prime}}$ for $m \neq m^{\prime}$ and solve

$$
\frac{\partial}{\partial x} \mu_{j}\left(x, t_{\mathfrak{g}}\right)=-2 i \frac{y\left(\hat{\mu}_{j}\left(x, t_{\mathfrak{g}}\right)\right)}{\prod_{\ell \neq j}^{\mathfrak{g}}\left(\mu_{j}\left(x, t_{\mathfrak{g}}\right)-\mu_{\ell}\left(x, t_{\mathfrak{g}}\right)\right)},
$$

$$
\frac{\partial}{\partial t_{\mathfrak{g}}} \mu_{j}\left(x, t_{\mathfrak{g}}\right)=\frac{\partial}{\partial x} \mu_{j}\left(x, t_{\mathfrak{g}}\right) \frac{1}{16 Q^{1 / 2}} \prod_{\ell \neq j}^{\mathfrak{g}} \mu_{\ell}\left(x, t_{\mathfrak{g}}\right), \quad j=1, \ldots, \mathfrak{g},
$$

with $Q=\prod_{m=1}^{2 \mathfrak{g}} E_{m}$ and $R_{2 \mathfrak{g}+1}(z)=z \prod_{m=1}^{2 \mathfrak{g}}\left(z-E_{m}\right)$. Define

$$
\begin{gathered}
u\left(x, t_{\mathfrak{g}}\right)=i \ln \left(Q^{-1 / 2} \prod_{j=1}^{\mathfrak{g}} \mu_{j}\left(x, t_{\mathfrak{g}}\right)\right), \\
V\left(x, t_{\mathfrak{g}}\right)=\sum_{m=0}^{2 \mathfrak{g}} E_{m}-2 \sum_{j=1}^{\mathfrak{g}} \mu_{j}\left(x, t_{\mathfrak{g}}\right) .
\end{gathered}
$$

Then $u$ and $V$ satisfy the sG equation and gth KdV equation, respectively, that is,

$$
4 u_{x, t_{\mathfrak{g}}}=\sin (u), \quad \operatorname{KdV}_{\mathfrak{g}}(V)=0
$$

for the following choice of $\tilde{c}_{\ell}$,

$\tilde{c}_{0}=1, \quad \tilde{c}_{1}=\frac{(-1)^{\mathfrak{g}-1}}{16 Q^{1 / 2}}-c_{1}(\underline{E}), \quad \tilde{c}_{\ell}=-\sum_{p=0}^{\ell-1} \tilde{c}_{p} c_{\ell-p}(\underline{E}), \quad \ell=2, \ldots, \mathfrak{g}$.

The isomorphism between algebro-geometric $\mathrm{KdV}_{\mathfrak{g}}$ and $\mathrm{sG}$ equations is of course well-known and has been discussed, for instance, in [5], [6].

$A K N S$ and $T l$. Pick $E_{0}, \ldots, E_{2 g+1} \in \mathrm{C}, E_{m} \neq E_{m^{\prime}}$ for $m \neq m^{\prime}$ and solve

$$
\frac{\partial}{\partial x} \mu_{j}\left(x, t_{r}\right)=-2 i \frac{y\left(\hat{\mu}_{j}\left(x, t_{r}\right)\right)}{\prod_{\ell \neq j}^{\mathfrak{g}}\left(\mu_{j}\left(x, t_{r}\right)-\mu_{\ell}\left(x, t_{r}\right)\right)},
$$

$$
\frac{\partial}{\partial t_{r}} \mu_{j}\left(x, t_{r}\right)=-\frac{\partial}{\partial x} \mu_{j}\left(x, t_{r}\right) \sum_{n=(r-\mathfrak{g}) \vee 0}^{r} d_{n} \Phi_{n}^{(j)}\left(\underline{\mu}\left(x, t_{r}\right)\right), \quad j=1, \ldots, \mathfrak{g}
$$


DUBROVIN EQUATIONS AND INTEGRABLE SYSTEMS ON HYPERELLIPTIC . . 121

and

$$
\frac{\partial}{\partial x} v_{j}\left(x, t_{r}\right)=-2 i \frac{y\left(\hat{v}_{j}\left(x, t_{r}\right)\right)}{\prod_{\ell \neq j}^{g}\left(v_{j}\left(x, t_{r}\right)-v_{\ell}\left(x, t_{r}\right)\right)},
$$

$$
\frac{\partial}{\partial t_{r}} v_{j}\left(x, t_{r}\right)=-\frac{\partial}{\partial x} v_{j}\left(x, t_{r}\right) \sum_{n=(r-\mathfrak{g}) \vee 0}^{r} d_{n} \Phi_{n}^{(j)}\left(\underline{v}\left(x, t_{r}\right)\right), \quad j=1, \ldots, \mathfrak{g},
$$

where $R_{2 \mathfrak{g}+2}(z)=\prod_{m=0}^{2 \mathfrak{g}+1}\left(z-E_{m}\right)$ and $d_{n} \in \mathrm{C}$. Define

$$
\begin{aligned}
& \frac{p_{x}\left(x, t_{r}\right)}{p\left(x, t_{r}\right)}=i \sum_{m=0}^{2 \mathfrak{g}+1} E_{m}-2 i \sum_{j=1}^{\mathfrak{g}} v_{j}\left(x, t_{r}\right), \\
& \frac{q_{x}\left(x, t_{r}\right)}{q\left(x, t_{r}\right)}=-i \sum_{m=0}^{2 \mathfrak{g}+1} E_{m}+2 i \sum_{j=1}^{\mathfrak{g}} \mu_{j}\left(x, t_{r}\right)
\end{aligned}
$$

and

$$
\begin{aligned}
a\left(n, t_{r}\right)^{2}=\frac{1}{2} \sum_{j=1}^{\mathfrak{g}} & \frac{y\left(\hat{\mu}_{j}\left(n, t_{r}\right)\right)}{\prod_{k \neq j}^{\mathfrak{g}}\left(\mu_{j}\left(n, t_{r}\right)-\mu_{k}\left(n, t_{r}\right)\right)} \\
& \quad-\frac{1}{4} b\left(n, t_{r}\right)^{2}-\frac{1}{4} \sum_{j=1}^{\mathfrak{g}} \mu_{j}\left(n, t_{r}\right)^{2}+\frac{1}{8} \sum_{m=0}^{2 \mathfrak{g}+1} E_{m}^{2},
\end{aligned}
$$

$$
b\left(n, t_{r}\right)=-\frac{1}{2} \sum_{m=0}^{2 \mathfrak{g}+1} E_{m}+\sum_{j=1}^{\mathfrak{g}} \mu_{j}\left(n, t_{r}\right)
$$

Then $(p, q)$ and $(a, b)$ satisfy the $r$ th AKNS equation and the $r$ th Toda lattice $(\mathrm{Tl})$ equation, respectively, that is,

$$
\operatorname{AKNS}_{r}(p, q)=0, \quad \mathrm{Tl}_{r}(a, b)=0
$$

for the same choice of $c_{\ell}, \ell=1, \ldots, r$ in both equations (6.10) (depending on the choice of $d_{n}$ in (6.6b), (6.7b)).

Remark 6.1. These examples provide interesting connections between the $\mathrm{KdV}_{\mathrm{g}}$ and sG equation (where $N$ is even and $0 \in\left\{E_{m}\right\}_{m=0, \ldots, N}$ ), and AKNS and Toda hierarchies (where $N$ is odd), respectively, and illustrate the fundamental role of the Dubrovin equations as the common underlying principle for 
hierarchies of soliton equations. In particular, our approach establishes an isomorphism between the classes of algebro-geometric solutions of these pairs of integrable systems. Indeed, once the hyperelliptic curve $\mathscr{K}_{\mathfrak{g}}$ is fixed, algebrogeometric solutions of the $\mathrm{KdV}_{\mathrm{g}}$ and sG (respectively, algebro-geometric solutions of the $r$ th AKNS and $r$ th Tl equation) are just certain symmetric functions (i.e., "trace" relations) of the solutions $\mu_{1}\left(x, t_{\mathfrak{g}}\right), \ldots, \mu_{\mathfrak{g}}\left(x, t_{\mathfrak{g}}\right)$ (resp. $\left.\mu_{1}\left(x, t_{r}\right), \ldots, \mu_{\mathfrak{g}}\left(x, t_{r}\right), v_{1}\left(x, t_{r}\right), \ldots, v_{\mathfrak{g}}\left(x, t_{r}\right)\right)$ of the corresponding Dubrovin equations on $\mathscr{K}_{\mathrm{g}}$. Analogous considerations apply to the nonlinear Schrödinger equation and the (continuum) Heisenberg chain (see, e.g., [22]).

\section{Appendix A. Lagrange interpolation formulas}

In the following we suppress the $\left(x, t_{r}\right)$-dependence as it will be of no importance in this appendix.

Fix $\mathfrak{g} \in \mathbb{N}$ and recall that $F_{\mathfrak{g}}(z)=\prod_{j=1}^{\mathfrak{g}}\left(z-\mu_{j}\right)$ implies $F_{\mathfrak{g}}^{\prime}\left(\mu_{k}\right)=$ $\prod_{\substack{j=1 \\ j \neq k}}^{\mathfrak{g}}\left(\mu_{k}-\mu_{j}\right)$ (with $\left.F_{\mathfrak{g}}^{\prime}=\partial F_{\mathfrak{g}} / \partial z\right)$. The general form of Lagrange's interpolation theorem then reads as follows. (For convenience of the reader we supply its proof even though the result is well-known.)

Theorem A.1. Assume that $\mu_{1}, \ldots, \mu_{\mathfrak{g}}$ are $\mathrm{g}$ distinct complex numbers. Then

$$
\begin{aligned}
\sum_{j=1}^{\mathfrak{g}} \frac{\mu_{j}^{m-1}}{F_{\mathfrak{g}}^{\prime}\left(\mu_{j}\right)} \Phi_{k}^{(j)}(\underline{\mu})=\delta_{m, \mathfrak{g}-k}-\Psi_{k+1}(\underline{\mu}) \delta_{m, \mathfrak{g}+1} & \\
& m=1, \ldots, \mathfrak{g}+1, k=0, \ldots, \mathfrak{g}-1 .
\end{aligned}
$$

PRoOF. Let $C_{R}$ be a circle with center at the origin and radius $R$ that contains the zeros $\mu_{j}$ of the polynomial $F_{\mathrm{g}}$ and which is oriented clockwise. Cauchy's theorem then yields

$$
\text { (A.2) } \begin{aligned}
\frac{1}{2 \pi i} \oint_{C_{R}} d \zeta \frac{\zeta^{m-1}}{F_{\mathfrak{g}}(\zeta)(\zeta-z)}= & \frac{z^{m-1}}{F_{\mathfrak{g}}(z)}+\sum_{k=1}^{\mathfrak{g}} \frac{\mu_{k}^{m-1}}{F_{\mathfrak{g}}^{\prime}\left(\mu_{j}\right)\left(\mu_{j}-z\right)}, \\
& z \neq \mu_{1}, \ldots, \mu_{\mathfrak{g}}, \quad m=1, \ldots, \mathfrak{g}+1 .
\end{aligned}
$$

However, by letting $R \rightarrow \infty$ one infers that (A.3)

$$
\frac{1}{2 \pi i} \oint_{C_{R}} d \zeta \frac{\zeta^{m-1}}{F_{\mathfrak{g}}(\zeta)(\zeta-z)}=\lim _{R \rightarrow \infty} \frac{R^{m-1}}{F_{\mathfrak{g}}(R)}=\delta_{m, \mathfrak{g}+1}, \quad m=1, \ldots, \mathfrak{g}+1,
$$


which implies

$$
z^{m-1}-\sum_{k=1}^{\mathfrak{g}} \frac{\mu_{k}^{m-1} F_{\mathfrak{g}}(z)}{F_{\mathfrak{g}}^{\prime}\left(\mu_{j}\right)\left(z-\mu_{j}\right)}=F_{\mathfrak{g}}(z) \delta_{m, \mathfrak{g}+1} .
$$

Using the symmetric functions $\Psi_{j}$ one may write

$$
F_{\mathrm{g}}(z)=\sum_{j=0}^{\mathfrak{g}} z^{\mathfrak{g}-j} \Psi_{j}(\underline{\mu})
$$

and

$$
\frac{F_{\mathfrak{g}}(z)}{z-\mu_{j}}=\sum_{k=0}^{\mathfrak{g}-1} z^{\mathfrak{g}-1-k} \Phi_{k}^{(j)}(\underline{\mu}) .
$$

Expanding both sides of equation (A.4) in powers in $z$, using (A.5) on the right-hand side and (A.6) on the left-hand side, proves (A.1).

For use in the main text we finally observe the following equalities. Adding (A.5) to $\mu_{j}$ times (A.6) one finds

$$
F_{\mathfrak{g}}(z)+\mu_{j} \frac{F_{g}(z)}{z-\mu_{j}}=\sum_{k=0}^{\mathfrak{g}-1} z^{\mathfrak{g}-k-1}\left(\Psi_{k+1}+\mu_{j} \Phi_{k}^{(j)}\right)+z^{\mathfrak{g}} .
$$

However, one also has

$$
F_{\mathrm{g}}(z)+\mu_{j} \frac{F_{\mathrm{g}}(z)}{z-\mu_{j}}=z \frac{F_{\mathfrak{g}}(z)}{z-\mu_{j}}=\sum_{k=0}^{\mathfrak{g}-1} z^{\mathfrak{g}-k-1} \Phi_{k+1}^{(j)}+z^{\mathfrak{g}},
$$

using (A.6) and recalling $\Phi_{\mathfrak{g}}^{(j)}=0$. Thus, one concludes

$$
\Psi_{k+1}(\underline{\mu})+\mu_{j} \Phi_{k}^{(j)}(\underline{\mu})=\Phi_{k+1}^{(j)}(\underline{\mu}), \quad k=0, \ldots, \mathfrak{g}-1 .
$$

Finally, we will show

$$
\sum_{\ell=0}^{k} \mu_{j}^{\ell} \Psi_{k-\ell} \underline{(\mu)}=\Phi_{k}^{(j)}(\underline{\mu}), \quad k=0, \ldots, \mathfrak{g},
$$

by induction. Equation (A.10) clearly holds for $k=0$; next assume that

$$
\sum_{\ell=0}^{k-1} \mu_{j}^{\ell} \Psi_{k-1-\ell}=\Phi_{k-1}^{(j)}
$$


holds. Then

(A.12)

$$
\begin{aligned}
\sum_{\ell=0}^{k} \mu_{j}^{\ell} \Psi_{k-\ell} & =\Psi_{k}+\mu_{j} \sum_{\ell=1}^{k} \mu_{j}^{\ell-1} \Psi_{k-\ell}=\Psi_{k}+\mu_{j} \sum_{\ell=0}^{k-1} \mu_{j}^{\ell} \Psi_{k-1-\ell} \\
& =\Psi_{k}+\mu_{j} \Phi_{k-1}^{(j)}=\Phi_{k}^{(j)}
\end{aligned}
$$

using first the induction hypothesis and then (A.9).

AcKnowledgments. H. H. is indebted to the Department of Mathematics at the University of Missouri, Columbia for the great hospitality extended to him during his sabbatical 1996-97 when major parts of this work were done.

\section{REFERENCES}

1. Al'ber, S. I., Investigation of equations of Korteweg-de Vries type by the method of recurrence relations, J. London Math. Soc. 19 (1979), 467-480 (Russian).

2. Al'ber, S. I., On stationary problems for equations of Korteweg-de Vries type, Comm. Pure Appl. Math. 34 (1981), 259-272 .

3. Al'ber, S. J., Associated integrable systems, J. Math. Phys. 32 (1991), 916-922.

4. Al'ber, S. I. and Al'ber, M. S., Formalisme hamiltonien pour les solutions "finite zone" d'équations intégrables, C. R. Acad. Sc. Ser. I 301 (1985), 777-780 .

5. Al'ber, S. J. and Al'ber, M. S., Hamiltonian formalism for nonlinear Schrödinger equations and sine-Gordon equations, J. London Math. Soc. (2) 36 (1987), 176-192.

6. Al'ber, S. J. and Al'ber, M. S., Hamiltonian formalism for finite-zone solutions of non-linear integrable equations, in VIIIth International Congress on Mathematical Physics, M. Mebkhout and R. Sénéor (eds.), World Scientific, Singapore, 1987, pp. 447-462.

7. Al'ber, M. S., Luther, G. G., and Marsden, J. E., Complex billiard Hamiltonian systems and nonlinear waves, in Algebraic Aspects of Integrable Systems. In Memory of Irene Dorfman, A. S. Fokas and I. M. Gelfand (eds.), Birkhäuser, Boston, 1997, pp. 1-16.

8. Belokolos, E. D., Bobenko, A. I., Enol'skii, V. Z., Its, A. R., and Matveev, V. B., AlgebroGeometric Approach to Nonlinear Integrable Equations, Springer, Berlin, 1994.

9. Birnir, B., Complex Hill's equation and the complex periodic Korteweg-de Vries equations, Comm. Pure Appl. Math. 39 (1986), 1-49.

10. Birnir, B., Singularities of the complex Korteweg-de Vries flows, Comm. Pure Appl. Math. 39 (1986), 283-305.

11. Bulla, W., Gesztesy, F., Holden, H., and Teschl, G., Algebro-geometric quasi-periodic finitegap solutions of the Toda and Kac-van Moerbeke hierarchies, Mem. Amer. Math. Soc. 135 (1998), No. 641, 1-79.

12. Burchnall, J. L. and Chaundy, T. W., Commutative ordinary differential operators, Proc. London Math. Soc. Ser. 2, 21 (1923), 420-440.

13. Burchnall, J. L. and Chaundy, T. W., Commutative ordinary differential operators, Proc. Roy. Soc. London Ser. A 118 (1928), 557-583.

14. Burchnall, J. L. and Chaundy, T. W., Commutative ordinary differential operators II. The identity $P^{n}=Q^{m}$, Proc. Roy. Soc. London Ser. A 134 (1932), 471-485.

15. De Concini, C. and Johnson, R. A., The algebraic-geometric AKNS potentials, Ergod. Theory Dynam. Systems 7 (1987), 1-24.

16. Date, E. and Tanaka, S., Periodic multi-soliton solutions of Korteweg-de Vries equation and Toda lattice, Progr. Theoret. Phys. Suppl. 59 (1976), 107-125. 
17. Date, E. and Tanaka, S., Analogue of inverse scattering theory for the discrete Hill's equation and exact solutions for the periodic Toda lattice, Progr. Theoret. Phys. 56 (1976), 457-465.

18. Dickey, L. A., Soliton Equations and Hamiltonian Systems, World Scientific, Singapore, 1991.

19. Dickson, R., Gesztesy, F., and Unterkofler, K., A new approach to the Boussinesq hierarchy, Math. Nachr. 198 (1999), 51-108.

20. Dickson, R., Gesztesy, F., and Unterkofler, K., Algebro-geometric solutions of the Boussinesq hierarchy, Rev. Math. Phys. 11 (1999), 823-879.

21. Dubrovin, B. A., Periodic problems for the Korteweg-de Vries equation in the class of finite band potentials, Functional Anal. Appl. 9 (1975), 215-223.

22. Elgin, J. N., Comments on the periodic nonlinear Schrödinger equation, in Nonlinear Evolution Equations: Integrability and Spectral Methods, A. Degasperis, A. P. Fordy, and M. Lakshmanan (eds.), Manchester University Press, Manchester, 1990, pp. 279-285.

23. Ercolani, N. M. and Flaschka, H., The geometry of the Hill equation and of the Neumann system, Philos Trans. Roy. Soc. London Ser. A 315 (1985), 405-422.

24. Farkas, H. M. and Kra, I., Riemann Surfaces, 2nd ed., Springer, New York, 1992.

25. Flaschka, H., On the inverse problem for Hill's equation, Arch. Rational Mech. Anal. 59 (1975), 293-309.

26. Gel'fand, I. M. and Dikii, L. A., Asymptotic behavior of the Resolvent of Sturm-Liouville equations and the algebra of the Korteweg-de Vries equations, Russ. Math. Surveys 30:5 (1975), 77-113.

27. Gel'fand, I. M. and Dikii, L. A., Integrable nonlinear equations and the Liouville theorem, Functional Anal. Appl. 13 (1979), 6-15.

28. Gesztesy, F. and Holden, H., A combined sine-Gordon and modified Korteweg-de Vries hierarchy and its algebro-geometric solutions, in Differential Equations and Mathematical Physics, Proceedings of an International Conference held at the University of Alabama at Birmingham, March 16-20, 1999, Rudi Weikard and Gilbert Weinstein (eds.), International Press, Boston 2000, pp. 133-173.

29. Gesztesy, F. and Holden, H., Soliton Equations and their Algebro-Geometric Solutions, Cambridge University Press, Cambridge, 2002, to appear.

30. Gesztesy, F. and Ratneseelan, R., An alternative approach to algebro-geometric solutions of the AKNS hierarchy, Rev. Math. Phys. 10 (1998), 345-391.

31. Gesztesy, F., Ratnaseelan, R., and Teschl, G., The KdV hierarchy and associated trace formulas, in proceedings of the International Conference on Applications of Operator Theory, I. Gohberg, P. Lancaster, P. N. Shivakumar (eds.), Operator Theory: Advances and Applications, Vol. 87, Birkhäuser, 1996, pp. 125-163.

32. Griffiths, P. A., Linearizing flows and a cohomological interpretation of Lax equations, Amer. J. Math. 107 (1985), 1445-1483.

33. Jacobi, C. G. T., Über eine neue Methode zur Integration der hyperelliptischen Differentialgleichungen und über die rationale Form ihrer vollständigen algebraischen Integralgleichungen, J. Reine Angew. Math. 32 (1846), 220-226.

34. Levitan, B. M., Inverse Sturm-Liouville Problems, VNU Science Press, Utrecht, 1987.

35. Marchenko, V. A., Sturm-Liouville Operators and Applications, Birkhäuser, Basel, 1986.

36. McKean, H. P., Variation on a theme of Jacobi, Comm. Pure Appl. Math. 38 (1985), 669-678.

37. van Moerbeke, P., The spectrum of Jacobi matrices, Invent. Math. 37 (1976), 45-81.

38. Mumford, D., Tata Lectures on Theta II, Birkhäuser, Boston, 1984.

39. Previato, E., Seventy years of spectral curves: 1923-1993, in Integrable Systems and Quantum Groups, M. Francaviglia and S. Greco (eds.), Lecture Notes in Math. 1620 (1996), 419481.

40. Schimming, R., An explicit expression for the Korteweg-de Vries hierarchy, Acta Appl. Math. 39 (1995), 489-505.

41. Toda, M., Theory of Nonlinear Lattices, 2nd enlarged ed., Springer, Berlin, 1989. 
42. Wilson, G., Algebraic curves and soliton equations, in Geometry Today, E. Arbarello, C. Procesi, and E. Strickland (eds.), Birkhäuser, Boston, 1985, pp. 303-329.

43. Nijhoff, F. W., Discrete Dubrovin equations and separation of variables for discrete systems, Chaos, Solitons and Fractals 11 (2000), 19-28.

DEPARTMENT OF MATHEMATICS

UNIVERSITY OF MISSOURI

COLUMBIA, MO 65211

USA

E-mail: fritz@math.missouri.edu
DEPARTMENT OF MATHEMATICAL SCIENCES

NORWEGIAN UNIVERSITY OF SCIENCE AND TECHNOLOGY NO-7491 TRONDHEIM

NORWAY

E-mail: holden@math.ntnu.no 